\title{
Trabajo colaborativo y software social. apreciaciones de los estudiantes universitarios alicantinos respecto al trabajo colaborativo mediante el software social
}

\section{Extracto:}

Las tecnologías de la información y la comunicación (TIC) han revolucionado la educación, promoviendo el desarrollo del trabajo colaborativo a través del uso de las herramientas de la Web 2.0. En este sentido, cobran especial importancia los servicios de redes sociales. No obstante, el establecimiento en el ámbito universitario de una metodología colaborativa mediante las herramientas TIC no puede ser eficaz sin una buena predisposición por parte del estudiantado. En consecuencia, esta investigación pretende conocer las apreciaciones sobre el trabajo colaborativo y las redes sociales de los alumnos del grado en Educación Infantil de la Universidad de Alicante. Los objetivos tratan de saber las actitudes del alumnado sobre el trabajo colaborativo y las redes sociales, conocer su nivel de experiencia en relación a estas herramientas, así como analizar su interés para introducirlas en el contexto académico. El instrumento de recogida de datos es un cuestionario. Se concluye que los discentes muestran una actitud positiva para trabajar en equipo y que están interesados en aprender a manejar ciertos servicios sociales.

Palabras clave: tecnologías de la información y la comunicación (TIC), trabajo cola-

\footnotetext{
${ }^{1}$ L. Ramos Marcillas, estudiante de la Universidad a Distancia de Madrid (UDIMA) y maestra de Educación Primaria en el Colegio de Educación Infantil y Primaria (CEIP) Virgen del Pilar (Pilar de la Horadada, Alicante).

2 A. Rodríguez Ruibal, profesor doctor de la Universidad a Distancia de Madrid (UDIMA).
} 


\section{Collaborative work and social networks! assessment of university students from Alicante about collaborative work through social networks}

\section{Abstract:}

The information and communication technologies (ICT) has revolutionized education, promoting the development of collaborative work through the use of Web 2.0 tools. In this vein, social networking services gain a special importance. However, the establishment of a collaborative methodology in the university through the use of ICT tools cannot be effective without a good predisposition of students. So, the present investigation aims to know students' perceptions about collaborative work and social networks. Specifically, the learners are studying Pre-School Education degree at Alicante's University. The goals are focused on knowing students' attitudes about collaborative work and social networks, knowing their level of experience about these tools, and also analyzing their interest in introducing these tools in the academic world. The instrument for data gathering is a questionnaire. It concludes that the students show a positive attitude towards team work and they are interested in learning to handle some social media.

Keywords: information and communication technologies (ICT), collaborative work, social networks, students' perceptions.

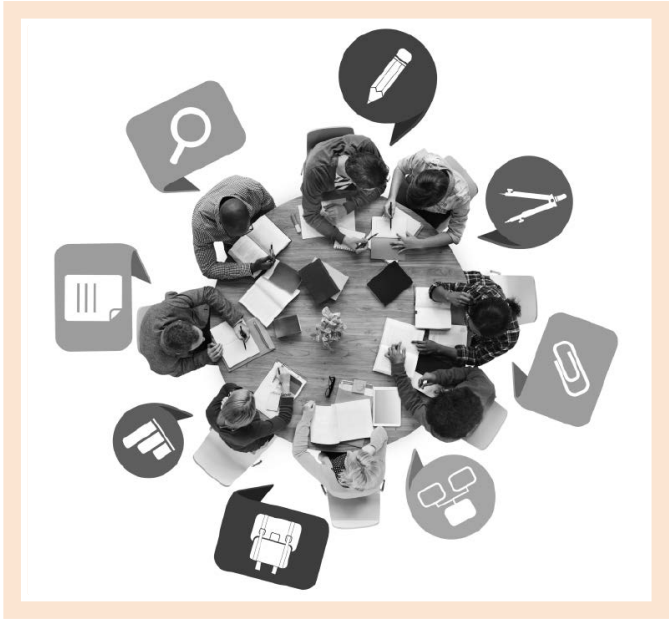

\section{INTRODUCCIÓN}

\subsection{Motivación}

El término TIC, tal y como argumentan Sicilia y García (2012), «se utiliza habitualmente para definir el conjunto de principios científicos y tecnológicos que permiten desarrollar, potenciar y mejorar el uso y manejo de la información, así como la comunicación de la misma entre distintos sistemas».

En la actualidad se ha producido una innovación disruptiva en el ámbito de la educación, ya que la aparición de las TIC ha transformado las formas de pensar, aprender, interaccionar y vivir. Parafraseando a Cabero (2009), la tecnología repercute en que los jóvenes procesen la información de forma distinta, formen redes sociales con otras reglas de juego y aborden el proceso de formación e instrucción desde otras perspectivas.

Dentro de la revolución de las herramientas TIC, cabe hacer especial hincapié en los servicios de redes sociales. Tal y como afirman Sixto y Túñez (2012), «la importancia y la repercusión que han adquirido las redes sociales en los últimos años son indiscutibles». Así pues, estos autores señalan que «Facebook lidera el ranking mundial, de tal forma que 3 de cada 10 usuarios que acceden a internet en algún lugar del planeta (35,58\%) visitan este sitio web. [Esto] convierte a la red social en la segunda página más visitada del mundo». 
En líneas generales, los servicios de redes sociales son aplicaciones que ponen en contacto a las personas a través de internet (De Haro, 2010). En este sentido, Castaño et al. (2008), citados por Sixto y Túñez (2012), identifican seis características diferenciales de las redes sociales:

«(...) 1) son dinámicas, puesto que los contenidos se actualizan de forma constante; 2) son colaborativas, pues se elaboran por un grupo de personas; 3 ) son simples e intuitivas; 4) pueden utilizarse sin necesidad de instalar nada en el ordenador, ya que la web es la plataforma; 5) poseen un entorno amigable e interactivo; y 6) los usuarios tienen capacidad de gestionar qué, cuándo y cómo publicar».

En cuanto a las ventajas que aportan las redes sociales, cabe mencionar las señaladas por García Orosa (2009), citada por García y Alonso (2014):

«El dinamismo, la confluencia de personas con intereses comunes y la facilidad para la difusión de un determinado mensaje».

Asimismo, las redes sociales también aportan múltiples beneficios al contexto académico, como pueden ser los apuntados por García (2010), citada por Fernández (2013):

«(...) a) motivación en el proceso educativo, ante la confianza por parte del profesor de cierta autonomía y libertad de acción (...);

\footnotetext{
El término TIC, tal y como argumentan Sicilia y García (2012), use utiliza habitualmente para definir el conjunto de principios científicos y tecnológicos que permiten desarrollar, potenciar y mejorar el uso y manejo de la información, así como la comunicación de la misma entre distintos sistemas»
}

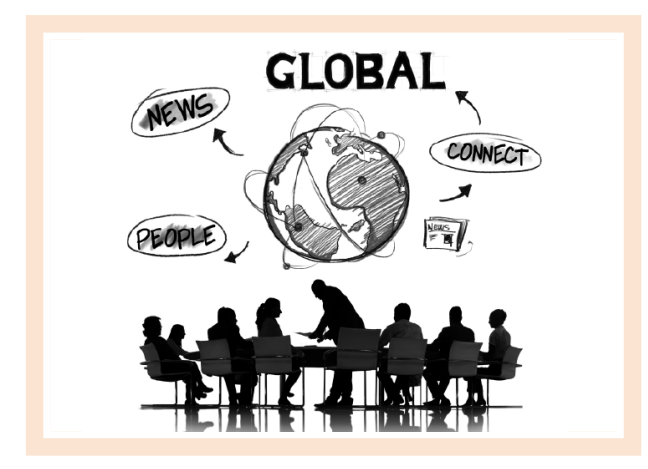

b) estimulación del trabajo autónomo, en interacción con los demás; c) facilidad e incentivación de la comunicación y del conocimiento mutuo de los participantes. Lo que también redunda en una ayuda a la labor docente, al poder conocer el profesor el perfil de su alumnado para la elaboración de la materia y al poder mantener un contacto virtual más ágil y continuo; d) favorece la retención de lo aprendido, ya que aprenden haciendo; e) práctica de la capacidad de expresión escrita y en el manejo de las TIC; f) gratuidad del servicio $(\ldots) ;$ g) prolongación del proceso de aprendizaje más allá del espacio físico y temporal del centro; h) incentivación del aprendizaje activo, en el que el alumnado se convierte en el agente de su propio aprendizaje y el profesor abandona el liderazgo como única fuente de conocimiento; i) revalorización del trabajo en equipo; j) posibilidad de integración de contenidos propios y de otros profesores de distintas procedencias y ubicaciones geográficas; k) estimulación de los progresos y logros mediante la opción de mandar "regalos virtuales" al alumnado, como medio de incentivación del proceso de aprendizaje; I) permite la creación de grupos especializados en algún tema específico dentro de la propia red, por lo que es posible realizar una sola red y aplicarla para varias asignaturas o varios grupos de alumnos».

Cabe destacar el fenómeno de las redes sociales como facilitadoras del aprendizaje colaborativo. 


\section{(...) siguiendo la postura de García} (2009), «el aprovechamiento de

\section{las herramientas que nos brinda}

la Web 2.0 (...) resulta una ventaja

altamente competitiva para

trabajar de forma colaborativa

en el aula, favoreciendo la

motivación y el interés de

los alumnos por su propio

aprendizaje»

En concreto, como señala García (2009), el aprendizaje colaborativo es el «intercambio y desarrollo de conocimiento en el seno de pequeños grupos de iguales, encaminados a la consecución de objetivos académicos». En cuanto a este aprendizaje, Fernández (2013) afirma que «se sustenta en el cognitivismo, rechazándose la observación pasiva, la repetición y la memorización». Así pues, algunas de las ventajas del trabajo colaborativo mencionadas por Martín-Moreno (2004), citado por García (2009), son:

«(...) a) el aprendizaje colaborativo incrementa la motivación de todos los integrantes del grupo hacia los objetivos y contenidos del aprendizaje; b) el aprendizaje que consigue cada individuo del grupo incrementa el aprendizaje del grupo y sus integrantes alcanzan mayores niveles de rendimiento académico; c) favorece una mayor retención de lo aprendido; d) promueve el pensamiento crítico (análisis, síntesis y evaluación de los conceptos), al dar oportunidades a sus integrantes de debatir los contenidos objeto de su aprendizaje; e) la diversidad de conocimientos y experiencias del grupo contribuye positivamente al proceso de aprendizaje, al tiempo que reduce la ansiedad que provocan las situaciones individuales de resolución de problemas».
Cabe destacar que Carrió (2006), citada por Fernández (2013), recoge algunos de los beneficios de las herramientas TIC y los grupos de colaboración, tales como:

«(...) a) facilitar el aprendizaje colaborativo, ya que se puede compartir información, mediante ficheros, contactar rápidamente, realizar foros de discusión, etc.; b) se puede realizar un seguimiento del progreso de los integrantes del grupo a través de las acciones que realizan y que automáticamente podemos seguir con las nuevas tecnologías (...); c) difundir las experiencias y poder contactar con otros grupos que realicen experiencias similares, compartiendo conocimientos y fuentes bibliográficas; $d$ ) investigar sobre distintos logros con otros grupos aunque estén en lugares muy distintos».

En concreto, Rendón y Ortega (2015) apuntan que la red social Facebook, desde una perspectiva colaborativa, facilita «un espacio virtual en el que los usuarios que comparten un objetivo común pueden publicar contenido, compartir ideas y opiniones, discutir sobre temas y organizar eventos». En este sentido, siguiendo la postura de García (2009), «el aprovechamiento de las herramientas que nos brinda la Web 2.0 (...) resulta una ventaja altamente competitiva para trabajar de forma colaborativa en el aula, favoreciendo la motivación y el interés de los alumnos por su propio aprendizaje».

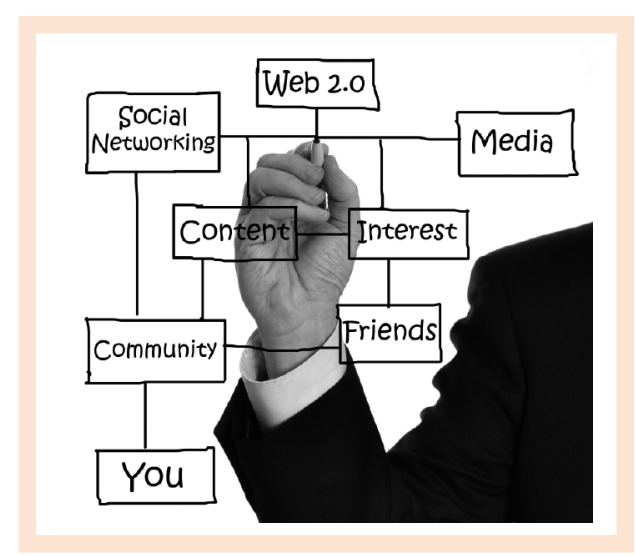




\subsection{Planteamiento del problema}

Dado el vínculo que existe entre las redes sociales y el trabajo en equipo, en el presente estudio se pretende investigar las percepciones del alumnado universitario en relación al aprendizaje colaborativo y las herramientas de software social. En este sentido, la implantación en el sistema académico superior de una metodología colaborativa a través de las herramientas de la Web 2.0 no podrá ser efectiva sin una buena predisposición por parte del alumnado. De este hecho deriva la importancia de conocer sus apreciaciones respecto a este fenómeno educativo.

Para llevar a cabo dicho trabajo, se toman como base las investigaciones de Cabero y Marín (2013 y 2014), las cuales se han realizado en el ámbito nacional e internacional. Estas investigaciones analizan las actitudes del alumnado universitario respecto a las redes sociales y el trabajo grupal en diversos países latinoamericanos (Venezuela, República Dominicana y Argentina) -investigación de 2013- y en diferentes ciudades españolas (Sevilla, Huelva, Córdoba y País Vasco) -investigación de 2014-.

En concreto, cabe resaltar las conclusiones a las que llegan Cabero y Marín (2013). En relación a las percepciones del alumnado respecto al software social y al trabajo en grupo, los autores afirman que «los estudiantes, independientemente del país latinoamericano, tienen una actitud positiva para el trabajo en grupo y consideran que este no repercute en resultados negativos para el aprendizaje». Además, Cabero y Marín (2013) también concluyen que «si los social media están llamando a las puertas de las instituciones de formación, el conocimiento que afirman tener nuestros alumnos sobre ellas, más sus percepciones para el trabajo en grupo, ofrecen altas expectativas para su incorporación a la formación». Por otra parte, el trabajo de Cabero y Marín (2014) pone de manifiesto que «los alumnos, independientemente de su universidad de procedencia, muestran altas percepciones para trabajar en grupo y de manera colaborativa».

Sin embargo, una investigación de este tipo no se ha realizado en ninguna de las universidades de las provincias de la Comunidad Valenciana. Por ello, la presente investigación se encuadra en la Universidad de Alicante. En concreto, se analizan las percepciones sobre esta temática de los alumnos del grado de Educación Infantil. En este sentido, este es un estudio ético, capaz de complementar y aportar una visión innovadora a las investigaciones mencionadas con anterioridad.

\subsection{Hipótesis de partida}

En relación con lo anteriormente expuesto, esta investigación pretende verificar la siguiente hipótesis:

«Los alumnos del grado de Educación Infantil de la Universidad de Alicante tienen actitudes positivas respecto al trabajo colaborativo en las redes sociales».

\subsection{Objetivos}

Entre los objetivos de esta investigación están los siguientes:

- Conocer las percepciones de los estudiantes universitarios en relación a las redes sociales y al trabajo colaborativo.

- Saber el nivel de experiencia que tienen los alumnos en las redes sociales.

- Analizar el interés de los discentes para introducir estas herramientas en el ámbito educativo.

\subsection{Metodología}

En cuanto a la metodología, se utiliza un cuestionario basado en el estudio de Cabero y Marín (2014), el cual, a su vez, ha sido extraído de la investigación realizada por Poellhuber, Anderson y Roy (2011). Dicho cuestionario consta de 55 ítems, los cuales están distribuidos en diversas dimensiones:

- Datos descriptivos.

- Preferencias de aprendizaje.

- Habilidades tecnológicas.

- Experiencia social del software.

- Software social para el aprendizaje. 


\section{TRABAJOS RELACIONADOS}

Múltiples son las investigaciones que han analizado las actitudes y los usos didácticos de las redes sociales en las diferentes etapas del ámbito educativo. Sin embargo, el ámbito más estudiado es el universitario. En este sentido, Martínez (2014) se centra en el papel de las redes sociales y de las TIC en la educación superior del siglo XXI. Algunas de las conclusiones que se pueden extraer de dicha investigación son las siguientes: la educación debe acomodarse a las exigencias actuales de la sociedad; la incidencia de las nuevas tecnologías en el proceso educativo está obligando a los actores de la educación a cambiar los procesos y las herramientas con los que se construye el conocimiento; hay que volver al para qué de la educación y no limitarse al cómo; y las TIC, en general, y las redes sociales, en particular, deben ser un apoyo para el proceso educativo, no un sinónimo de superficialidad.

Por otra parte, Espuny et al. (2011) reflexionan acerca de la actitud que sus propios estudiantes tienen con respecto al uso, al aprovechamiento didáctico y a las potencialidades que las redes sociales presentan en el ámbito educativo. Así pues, estos autores concluyen que sus alumnos «presentan una buena actitud como usuarios de las redes sociales. Los índices de conocimiento y uso de algunas de ellas son especialmente altos, lo cual pone de manifiesto la ausencia de un especial recelo que los lleve a no utilizarlas». Sin embargo, Espuny et al. (2011) declaran que sus estudiantes, los cuales no han utilizado estos servicios en el ámbito académico, "son susceptibles de utilizar las redes sociales en el contexto académico con provecho».

Otro estudio interesante referente al ámbito universitario es el realizado por Sánchez, Ruiz y Sánchez (2015). Estos autores investigan el empleo de las redes sociales por parte del alumnado universitario, analizando posibles malos hábitos y usos problemáticos de las mismas. En consecuencia, Sánchez, Ruiz y Sánchez (2015) concluyen que su alumnado «posee una buena predisposición al uso de las redes sociales. Los porcentajes de conocimiento y empleo de las mismas son lo suficientemente altos, indicando que están plenamente integradas en su día a día». Pero estos autores «no (...) han constatado problemas de dependencia ni de alteración de las conductas habituales en los encuestados». Además, afirman que «una de las principales causas por las que los estudiantes no relacionan el empleo de las redes sociales con el ámbito académico es debido (...) a que las instituciones educativas no otorgan a estas el papel fundamental que poseen en el día a día de los alumnos».

Por lo que respecta a los perfiles de uso de las redes sociales en el alumnado universitario, cabe destacar la investigación realizada por Prendes, Gutiérrez y Castañeda (2015). Estos autores analizan el uso que los estudiantes universitarios hacen de este tipo de herramientas, confirmando que «las redes sociales forman parte de las herramientas básicas y habituales de comunicación entre los jóvenes de [su] universidad». Asimismo, prosiguen que «se utilizan para fines eminentemente lúdicos». En consecuencia, los autores concluyen que «los estudiantes universitarios (...) no son conscientes del amplio abanico de posibilidades que (...) las redes sociales pueden ofrecer desde el punto de vista profesional». Es por ello que los alumnos conocen Facebook y Twitter, pero hay un desconocimiento general de la red social profesional más importante de la actualidad: Linkedln.

En concreto, también hay que señalar algunas investigaciones que se centran en redes sociales específicas. Por ejemplo, Bicen y Cavus (2011) investigan el uso que hacen los estudiantes de Facebook. Según los resultados, «students spend a significant amount of their times using the Facebook. (...) Students increase their knowledge and communication skills by sharing photographs, links that they like, and news with their friends».

En esta misma línea de investigación, Towner y Muñoz (2011) tratan de examinar las actitudes hacia el uso de Facebook como una herramienta propia del ámbito académico. La principal conclusión obtenida dice así: «Students (...) are less accepting of using Facebook for informal and formal teaching practices». De este modo, «most students are not open to personal communication with their instructor through Facebook». Otra conclusión es la que sigue: «Faculty are also reluctant to adopt Facebook for educational practices». 
En última instancia, cabe resaltar diversas investigaciones que abordan el estudio de las redes sociales y el trabajo colaborativo. En este sentido, Garrigós et al. (2010) realizan un experimento en la asignatura Base de Datos Avanzadas de la carrera Ingeniería Informática para analizar el impacto de Facebook como herramienta colaborativa en la docencia universitaria. Las conclusiones a las que llegan estos autores son:

«(...) 1) la mejora en la comunicación [a través del uso de Facebook] se produce más significativamente entre profesor-alumno, ya que la relación entre alumnos ya existía previamente; 2) entre los alumnos preocupa el tema de usar una herramienta de ocio en un ámbito educativo, puesto que ven mermada su privacidad; y 3) las redes sociales actuales carecen de determinadas herramientas que son básicas para la docencia (como el repositorio de documentos)».

Finalmente, García (2009) realiza un estudio intensivo de Facebook como herramienta de aprendizaje colaborativo. Así pues, la autora, en primer lugar, explica qué es el trabajo colaborativo y cómo aplicarlo en la docencia universitaria. Posteriormente, García se centra en Facebook como una de las redes sociales que facilita la cooperación a través de la creación de grupos. Algunas de las acciones que se pueden realizar en los grupos de trabajo, una vez analizado este artículo, son el envío de mensajes al grupo completo, la muestra de la información del grupo, la publicación de fotos, la publicación de vídeos, la publicación de artículos relacionados a vínculos, la utilización del foro de discusión, la compartición del grupo para darse a conocer, la realización de invitaciones a otros miembros, el uso del muro, la creación de un evento, etc.

Una vez expuestas estas investigaciones, cabe concluir que la mayoría de ellas coinciden en que, a pesar de que las redes sociales forman parte de la vida diaria de los estudiantes, estos no muestran una actitud plenamente positiva hacia su aplicación en el ámbito académico. Esta percepción de los alumnos puede ser debida a diferentes aspectos: no han tenido experiencia usando las redes sociales en educación; no conocen las posibilidades de estos servicios; las instituciones educativas y el profesorado se muestran reacios a incluirlos en este ámbito, entre otros motivos.
No obstante, los beneficios de las redes sociales en el sector educativo han sido constatados, destacando la promoción de la comunicación y la colaboración. Es por ello que en la presente investigación se analizan las apreciaciones del alumnado universitario sobre las redes sociales y el trabajo colaborativo.

\section{(...) a pesar de que las redes sociales forman parte de la vida diaria de los estudiantes, estos no muestran una actitud plenamente positiva hacia su aplicación en el ámbito académico}

\section{DESARROLLO}

En relación al desarrollo de la investigación, cabe destacar la muestra seleccionada y el método utilizado. Por una parte, el tipo de muestreo empleado es el no probabilístico-incidental, lo que significa que el investigador selecciona intencionalmente la muestra, ya que es representativa para el objeto de estudio y tiene fácil acceso a la misma. En este sentido, la muestra ha sido de un total de 70 sujetos, los cuales son alumnos del grado en Educación Infantil en la Universidad de Alicante (estos datos son los ítems 1 y 2 del cuestionario).

Por otra parte, el enfoque metodológico es de tipo cuantitativo, ya que se ha utilizado un cuestionario. Tal y como se ha explicado con anterioridad, el cuestionario está basado en el estudio de Cabero y Marín (2014), el cual, a su vez, ha estado fundamentado por el realizado por Poellhuber, Anderson y Roy (2011). En líneas generales, la adaptación del instrumento ha supuesto la reformulación de algunas preguntas concretas. Así pues, el cuestionario consta de 55 ítems y está estructurado en diferentes dimensiones:

I. Datos descriptivos.

II. Preferencias de aprendizaje.

III. Habilidades técnicas tecnológicas.

IV. Experiencia social del software.

V. Software social para el aprendizaje. 
Cabe resaltar que el cuestionario se ha administrado en papel «presencialmente» en la Facultad de Educación de la Universidad de Alicante. A continuación, se describen las dimensiones y se analizan los resultados obtenidos en cada pregunta. Se pueden consultar los gráficos que muestran dichos resultados en el anexo final.

\section{Dimensión I. «Datos descriptivos»}

Pretende saber algunos datos personales básicos de los estudiantes encuestados (universidad, grado, curso, sexo, edad), así como algunos ítems vinculados a la disposición de un ordenador personal y de conexión a internet.

En cuanto al curso al que pertenecen los sujetos, cabe destacar que las asignaturas en las que se ha realizado este cuestionario son de carácter optativo, por lo que los alumnos pueden pertenecer a diferentes cursos académicos. En este caso, la mayoría de los encuestados (47) están en el segundo año de carrera. Sin embargo, solamente hay dos personas, tanto en el primer curso, como en el cuarto. Los encuestados restantes son de tercer curso (19).

En lo que respecta al sexo de los alumnos encuestados, el $80 \%$ de la muestra son mujeres (56), mientras que solamente han completado el cuestionario 14 hombres. Este dato demuestra la inclinación de las mujeres por la profesión de magisterio infantil.

Otro dato personal que ha sido analizado es la edad de los participantes que han realizado el cuestionario. En este sentido, predominan los estudiantes entre 21 y 24 años (39), seguidos de los que tienen entre 17 y 20 años (16). A continuación, un total de 10 alumnos tienen una edad comprendida entre 25 y 28 años (10). Finalmente, solo 5 discentes son mayores de 29 años.

Por otra parte, cabe señalar que el $100 \%$ de los encuestados disponen de un ordenador personal, ya sea de sobremesa o portátil, así como de conexión a internet (preguntas 6 y 7 ). Este hecho refleja cómo en la actualidad se está reduciendo la brecha digital, definida como la desigualdad de posibilidades que existen para acceder a la información, al conocimiento y a la educación a través de las TIC (Cabero, 2011).

Otro de los aspectos que se analizan en esta dimensión es si los alumnos han realizado formación a través de internet (pregunta 8). Hay que resaltar que 52 encuesta-

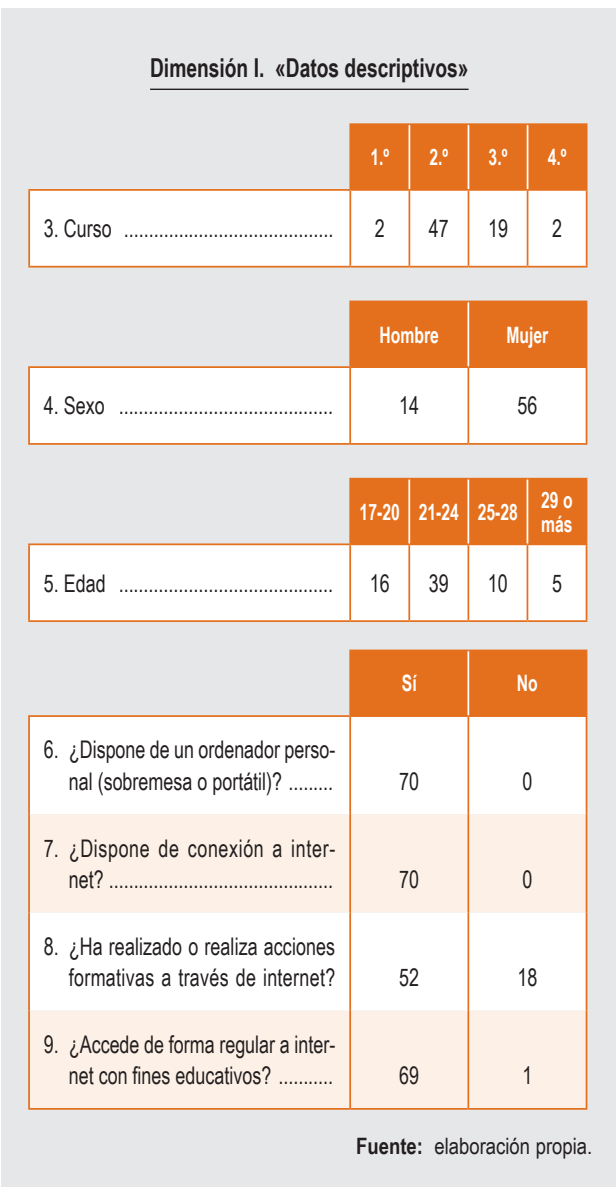

dos responden afirmativamente. Este hecho refleja una tendencia positiva a la realización de acciones formativas e-learning y/o b-learning. Además, dichas respuestas permiten inducir que la mayoría de los alumnos habrán hecho uso de herramientas de la Web 2.0.

La última pregunta de la dimensión 1 (pregunta 9) trata sobre el uso que los alumnos le dan a internet. En concreto, pretende conocer qué cantidad de estudiantes acceden regularmente a internet con fines educativos. La respuesta es significativa porque el $99 \%$ de los estudiantes responde de manera afirmativa. Los datos recogidos son similares a los expuestos por Cabero y Marín (2014) en su investigación. Así pues, según estos autores, «esto confirma que internet, como medio educativo, se ha extendido en las instituciones de educación superior». 


\section{Dimensión II. «Preferencias de aprendizaje»}

Está destinada a conocer cuáles son las preferencias de aprendizaje de los alumnos sobre el trabajo individual en contraposición al trabajo colaborativo. Este apartado tiene una construcción tipo Likert con cinco opciones de respuesta:

- TD (totalmente en desacuerdo).

- ED (en desacuerdo).

- $\mathbf{N}$ (ni de acuerdo ni en desacuerdo).

- DA (de acuerdo).

- TA (totalmente de acuerdo).

El análisis de esta segunda dimensión se realiza dividiendo las preguntas en dos grandes categorías: aprendizaje autónomo (preguntas de la 10 a la 16) y aprendizaje colaborativo (preguntas de la 17 a la 25). En cuanto a la primera categoría, se determina una ligera predisposición a anteponer el trabajo grupal sobre el trabajo individual. No obstante, se destaca que en la mayoría de las respuestas predomina la opción «ni de acuerdo, ni en desacuerdo».

Así pues, en la pregunta 10, el $49 \%$ de los encuestados están «totalmente en desacuerdo» o «en desacuerdo» a trabajar por su cuenta sin prestar atención a otros compañeros. Además, en el ítem 12, en total el $21 \%$ de los estudiantes están «de acuerdo» $(17 \%) 0$ «totalmente de acuerdo» (14\%) en que sus trabajos son de mayor calidad si los realizan de manera individual. Del mismo modo, en la pregunta 13 , el $39 \%$ de los discentes no creen que exista una relación entre el trabajo individual y la adquisición de capacidades para desenvolverse en el futuro; es por ello que señalan que están «en desacuerdo» 0 «totalmente en desacuerdo».

Por otra parte, en la pregunta 11 , el $37 \%$ de los alumnos están «de acuerdo» o «totalmente de acuerdo» en trabajar individualmente para avanzar con más rapidez.

\section{(...) se determina una ligera predisposición a anteponer el trabajo grupal sobre el trabajo individual}

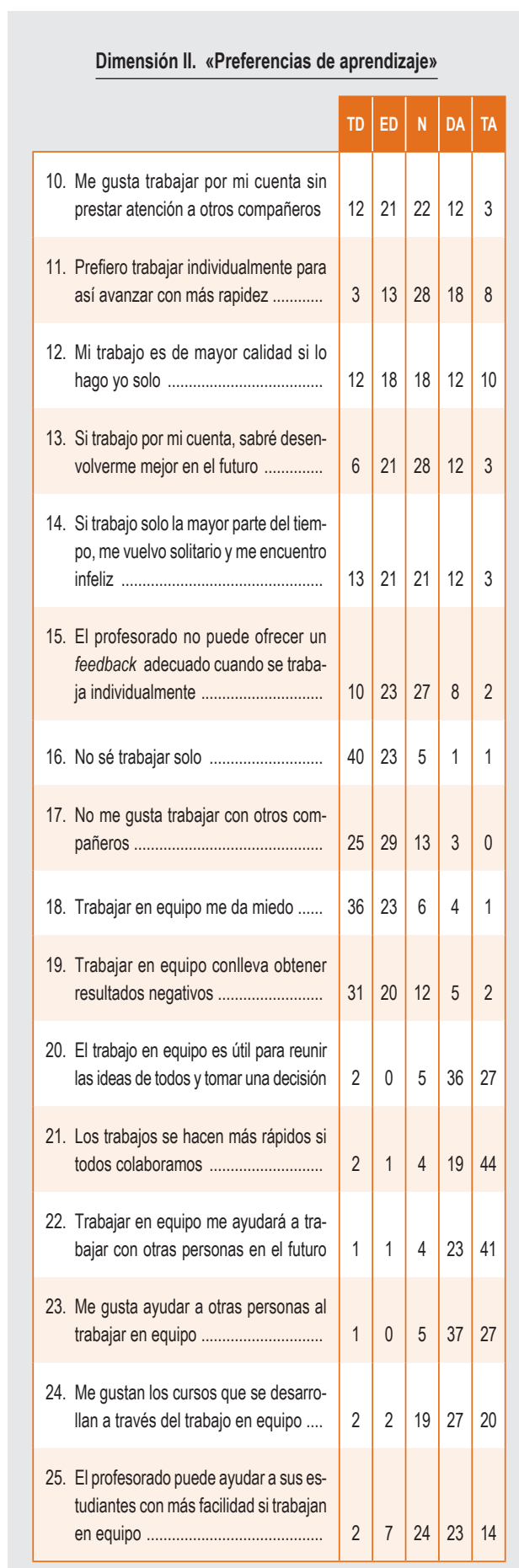

Fuente: elaboración propia 


\section{(...) es cierto que los estudiantes contemplan la posibilidad de realizar trabajos de forma colaborativa para potenciar ciertos aspectos de los mismos, por ejemplo, su calidad}

Asimismo, en la pregunta 14 , el $49 \%$ de los estudiantes no están de acuerdo en que el trabajo individual les cause sensación de solitud o tristeza. Otro aspecto importante es que el $47 \%$ de las personas que han realizado el cuestionario opinan que el docente sí puede ofrecer un feedback adecuado y eficaz cuando el trabajo de los alumnos es individual, ya que se muestran «totalmente en desacuerdo» (14\%) 0 "en desacuerdo» (33\%) con la afirmación 15. Finalmente, cabe destacar que el $90 \%$ de los encuestados está «totalmente en desacuerdo» o "en desacuerdo» en que no sabe trabajar de manera autónoma (pregunta 16).

En consecuencia, los datos muestran que el alumnado tiene las habilidades necesarias para trabajar de forma individual y tiene en cuenta algunas de sus ventajas, tales como la rapidez para desarrollar ciertas actividades de aprendizaje. Sin embargo, es cierto que los estudiantes contemplan la posibilidad de realizar trabajos de forma colaborativa para potenciar ciertos aspectos de los mismos, por ejemplo, su calidad. En este sentido, como señalan Cabero y Marín (2014) en su investigación, «los alumnos participantes son conscientes de que una de las condiciones de aprender y trabajar en el siglo XXI es el trabajo en grupo y colaborativo».

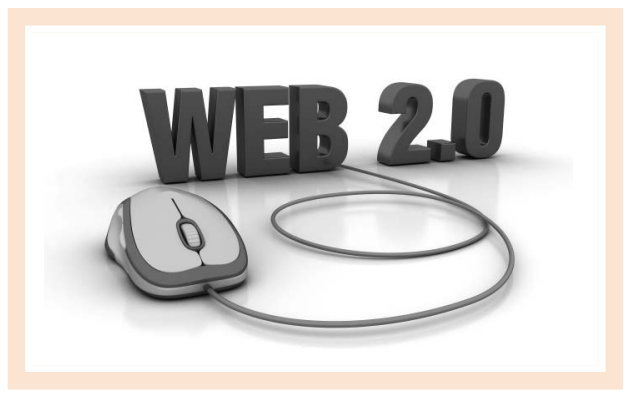

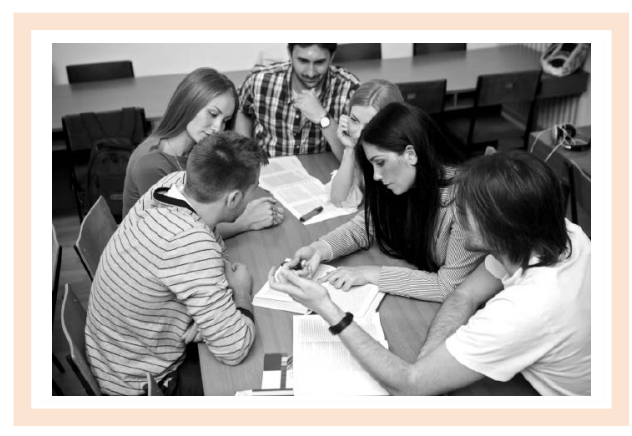

A continuación, en la categoría referida al trabajo colaborativo, cabe destacar que los resultados muestran una valoración muy positiva de este tipo de aprendizaje. En este sentido, en la pregunta 17 , el $77 \%$ de los encuestados están «totalmente en desacuerdo» 0 «en desacuerdo» en que no les gusta trabajar con otros compañeros. Asimismo, el $85 \%$ de los estudiantes también marcan estos valores en el ítem 18, el cual está vinculado a la sensación de miedo a la hora de trabajar en equipo. Además, la mayoría de los alumnos (73\%) señalan su disconformidad respecto a la afirmación «trabajar en equipo conlleva obtener resultados negativos», la cual se corresponde con la pregunta 19 del cuestionario.

En relación a la tendencia satisfactoria de los estudiantes respecto al trabajo colaborativo, hay que señalar que, en la pregunta 20 , un $90 \%$ de los encuestados consideran que el trabajo en equipo es útil para reunir las ideas de todos y tomar una decisión. En esta misma línea de pensamiento, en los ítems 21,22 y 23 , la mayoría de los alumnos ( $90 \%, 92 \%$ y $92 \%$, respectivamente) están «de acuerdo» 0 «totalmente de acuerdo» en que los trabajos se hacen más rápido si todos los miembros del grupo colaboran, en que trabajar en equipo les ayudará a trabajar con otras personas en el futuro y en que les gusta ayudar a otras personas al trabajar de forma colaborativa. En último lugar, la conformidad de los discentes no es tan rotunda en las preguntas 24 y 25 , ya que al $67 \%$ les gusta realizar cursos a través del trabajo colaborativo y el $53 \%$ piensa que el profesorado puede ayudar a los alumnos con más facilidad si trabajan en equipo.

Una vez analizada la dimensión II, es significativo resaltar que Cabero y Marín (2014) obtienen resultados 
similares a los expuestos anteriormente en su investigación llevada a cabo en diversas ciudades españolas. En este sentido, estos autores explican lo siguiente:

«El estudio señala con claridad que los estudiantes prefieren trabajar en grupo, como podemos observar en contestaciones a ítems como los que a continuación presentamos, donde el sumatorio de los porcentajes a las contestaciones "de acuerdo" y "totalmente de acuerdo" destaca sobre las demás:

- El trabajo en grupo es útil para reunir las ideas de todos y tomar una decisión $(88,9 \%)$.

- Los trabajos se hacen más rápidos si todos colaboramos $(85,9 \%)$.

- El trabajo con otros estudiantes me puede ayudar a aprender $(97,1 \%) »$.

Asimismo, los resultados también son parecidos a los obtenidos por Cabero y Marín (2013) en la investigación que llevaron a cabo en diversos países sudamericanos y en algunas ciudades españolas. Estos son los datos obtenidos por estos autores:

«El $89,73 \%$ indicó que sabía trabajar individualmente, ya que no estaban "totalmente en desacuerdo" o "en desacuerdo" con la frase "No sé trabajar solo", pero preferían trabajar en equipo, como podemos observar en las siguientes respuestas, donde la suma de porcentajes de las opciones "de acuerdo" y "totalmente de acuerdo" destaca sobre las demás:

- El trabajo en grupo es útil para reunir las ideas de todos y tomar una decisión $(86,55 \%)$

- Me gusta ser capaz de utilizar las ideas de otras personas, así como la mías propias $(85,90 \%)$.

- Los trabajos se hacen más rápido si todos colaboramos $(84,70 \%)$.

- El trabajo con otros estudiantes me puede ayudar a aprender $(97,15 \%)$.

\section{(...) en la categoría referida al trabajo colaborativo, (...) los resultados muestran una valoración muy positiva de este tipo de aprendizaje}

Esta preferencia también se observa en los ítems formulados negativamente, donde la suma de respuestas "totalmente en desacuerdo" y "en desacuerdo" prevalece sobre las demás:

- Trabajar en un grupo conlleva obtener resultados negativos (72,60\%).

- Trabajar en un grupo me da miedo (89,16\%).

- En una discusión de grupo, nunca se llega a conclusiones importantes $(74,96 \%)$.

- Me gusta trabajar por mi cuenta sin prestar atención a otros compañeros (75,68\%).

- No me gusta trabajar con otros compañe$\operatorname{ros}(82,47 \%)$.

(...) Por otra parte, se percibe que, para aprender y trabajar en el futuro, el trabajo en equipo es una condición necesaria:

- Si trabajo por mi cuenta, sabré desenvolverme mejor en el futuro, donde solamente el $27,81 \%$ se mostraban "de acuerdo" y "totalmente de acuerdo".

- Trabajar en grupo ahora me ayudará a trabajar con otras personas en el futuro, donde la gran mayoría $(91,60 \%)$ se mostraban "de acuerdo" y "totalmente de acuerdo"».

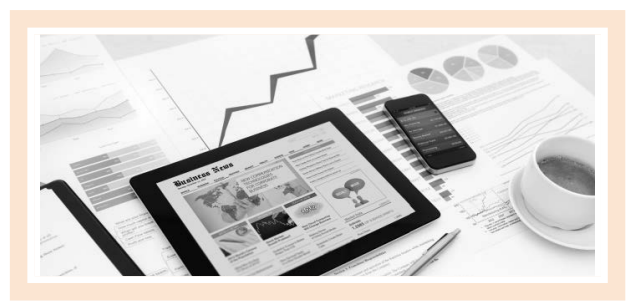




\section{Dimensión III. «Habilidades tecnológicas»}

Está enfocada a analizar las habilidades de los estudiantes con respecto al uso de las herramientas TIC. Este apartado tiene una construcción tipo Likert con cinco opciones de respuesta:

- TD (totalmente en desacuerdo).

- ED (en desacuerdo).

- $\mathbf{N}$ (ni de acuerdo ni en desacuerdo).

- DA (de acuerdo).

- TA (totalmente de acuerdo).

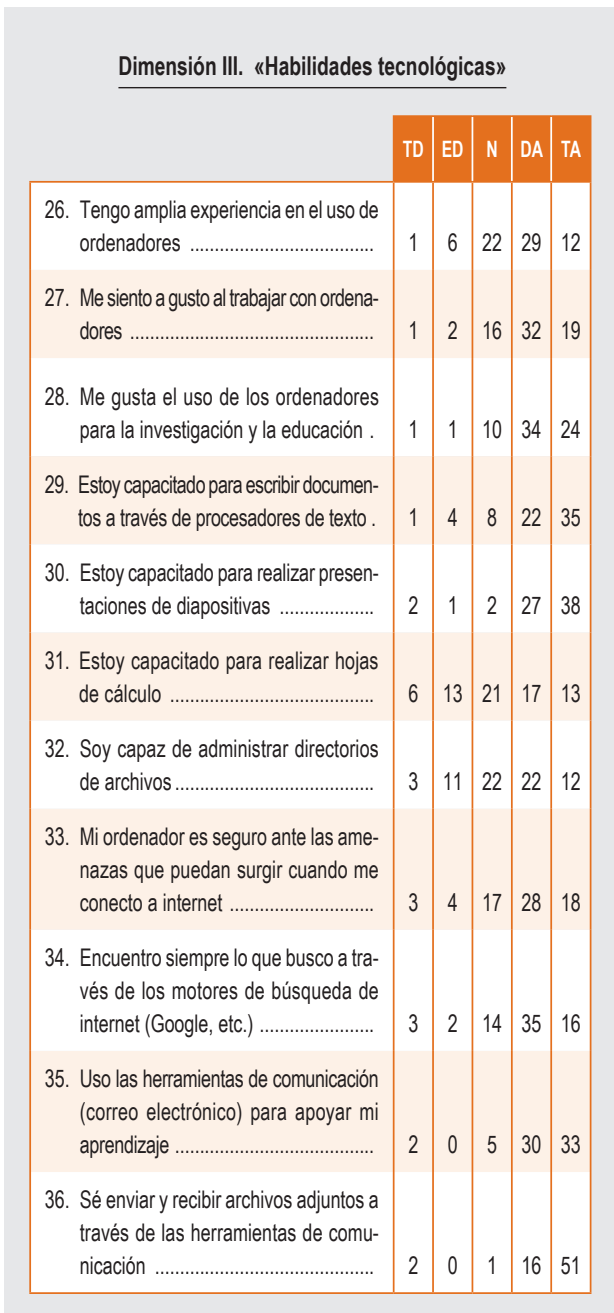

Fuente: elaboración propia
En primer lugar, cabe señalar que la mayoría de los participantes muestra una actitud positiva hacia el uso de los ordenadores. En este sentido, en la pregunta 26 , el $59 \%$ de los estudiantes están «de acuerdo» 0 «totalmente de acuerdo» en que tienen una amplia experiencia en el uso de este tipo de dispositivos. Además, en la pregunta 27 , el $73 \%$ de los encuestados se sienten cómodos a la hora de trabajar con ordenadores, ya que el $46 \%$ está «de acuerdo» y el $27 \%$ está «totalmente de acuerdo» con esta afirmación. Este hecho refleja cómo se está reduciendo la brecha generacional, ya que los jóvenes del siglo XXI no son reacios a la utilización de las nuevas tecnologías.

Por otra parte, es conveniente contemplar que uno de los usos que los alumnos les dan a los ordenadores está vinculado a la búsqueda de información referente a su carrera académica (por ejemplo, para realizar trabajos o elaborar temarios). En este sentido, en la pregunta 28 , casi la totalidad de los participantes (83\%) está «de acuerdo» 0 «totalmente de acuerdo» en que les gusta el uso de ordenadores para la investigación y la educación.

En relación a los paquetes ofimáticos, hay que destacar que existen diferencias entre la capacidad de los estudiantes para usar procesadores de texto, presentaciones de diapositivas y hojas de cálculo. Así pues, la rotundidad es clara en la habilidad para usar procesadores de texto, ya que en el ítem 29 , el cual versa sobre este aspecto, el $81 \%$ de los encuestados están «de acuerdo» o «totalmente de acuerdo». También, los alumnos muestran una gran capacidad para realizar presentaciones de diapositivas, puesto que en la pregunta 30 solo el $4 \%$ de los participantes están «totalmente en desacuerdo» 0 «en desacuerdo» en este sentido. Finalmente, las respuestas son variadas en la pregunta 31 , la cual está relacionada con la capacidad para realizar hojas de cálculo. Así, la respuesta más destacada es «ni de acuerdo, ni en desacuerdo», escogida por 21 estudiantes.

En cuanto a la organización del ordenador, cabe indicar que, en la pregunta 32, la cantidad de respuestas es similar en todos los valores. En consecuencia, no se puede confirmar con claridad que los estudiantes sepan administrar directorios de archivos. Otro aspecto destacable es que, en la pregunta 33 , el $66 \%$ de los estudiantes afirman estar "de acuerdo» 0 "totalmente de acuerdo» en que sus ordenadores son seguros ante las amenazas que pueden surgir cuando se conectan a internet. 
En otro orden de cosas, es importante mencionar que la mayoría de los participantes están capacitados para realizar búsquedas efectivas en internet a través de los motores de búsqueda disponibles. Así pues, el $73 \%$ de los estudiantes están «de acuerdo» 0 «totalmente de acuerdo» en el ítem 34. Por tanto, los alumnos son conscientes de que deben hacer uso de palabras clave para aumentar la precisión de sus búsquedas en la web.

Las últimas dos preguntas están relacionadas con las herramientas de comunicación on-line. Resulta significativo que el $90 \%$ de los encuestados utilizan herramientas de comunicación para apoyar su aprendizaje (pregunta 35). En este sentido, cabe destacar que las herramientas pueden ser tanto sincrónicas (el chat y la videoconferencia), como asincrónicas (el correo electrónico y los foros). Asimismo, los estudiantes poseen habilidades básicas para utilizar estas herramientas de comunicación, ya que, en el ítem 36, el $96 \%$ de los alumnos confirman que saben enviar y recibir adjuntos a través de este tipo de herramientas.

Tras analizar los resultados de la dimensión III, cabe destacar que son similares a los recogidos por Cabero y Marín (2014) en su investigación realizada exclusivamente en España. Según estos autores:

«Las percepciones que los alumnos tienen hacia las TIC son altamente positivas (...) [predominando] las opciones de respuestas "de acuerdo" y "completamente de acuerdo", (...) como ocurre en los siguientes casos:

- Me gusta el uso de los ordenadores para la investigación y la educación (87,1\%).

- Me gusta comunicarme con los demás mediante la comunicación asistida por ordenadores (por ejemplo, correo electrónico, mensajes de texto) como apoyo a mi aprendizaje $(83,2 \%)$.

- Me siento a gusto al trabajar con ordenadores $(79,2 \%)$.

Al mismo tiempo, los alumnos revelaron que se encontraban capacitados para realizar diferentes actividades:
- Sé cómo enviar y recibir mensajes y archivos adjuntos a través de diversas herramientas de comunicación (correo electrónico, mensajería instantánea, etc.) (95,6\%).

- Soy bueno para encontrar lo que busco cuando utilizo motores de búsqueda de internet (Google, Yahoo, etc.) (73,3\%).

- Estoy capacitado para escribir documentos a través de procesadores de texto (por ejemplo, utilizando el subrayado, las negriIlas, la creación, etc.) (92,3\%).

- Soy bueno en el uso de software de presentación (por ejemplo, Powerpoint) $(70,6 \%$ )».

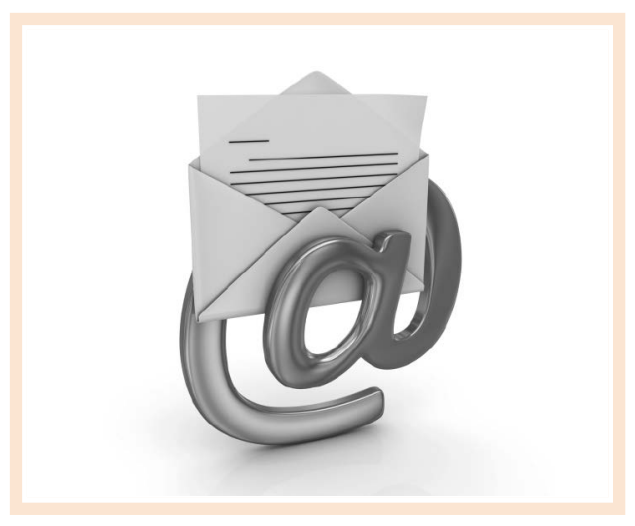

Por otra parte, los resultados también se corresponden con la investigación anterior de Cabero y Marín (2013), realizada en España y Sudamérica. En palabras de estos autores:

«Las percepciones hacia las TIC son positivas. En algunos casos, la suma de las opciones "de acuerdo" y "totalmente de acuerdo" supera el $80 \%$ :

- Me gusta el uso de los ordenadores para la investigación y la educación (90,67 \%).

- Me gusta comunicarme con los demás mediante la comunicación asistida por ordenador como apoyo a mi aprendizaje $(87,46 \%)$. 
- Me siento a gusto trabajando con ordenadores $(84,65 \%)$.

También informaron que se encontraban capacitados para realizar diferentes actividades, como podemos ver en los porcentajes obtenidos que sumaban las opciones de respuesta "de acuerdo" y "totalmente de acuerdo":

- Sé cómo enviar y recibir mensajes y archivos adjuntos a través de diversas herramientas de comunicación $(95,08 \%)$.

- Soy bueno para encontrar lo que busco cuando utilizo los motores de búsqueda de internet (Google, Yahoo, etc.) $(81,83 \%)$.

- Estoy capacitado para escribir documentos a través de procesadores de texto (por ejemplo, utilizando el subrayado, las negrillas, la creación de tablas, etc.) (90,63\%).

- Soy bueno en el uso de software de presentación (72,90\%)».

\section{Dimensión IV. «Experiencia social del software»}

Pretende conocer cuál es la experiencia del alumnado sobre las herramientas procedentes de la Web 2.0 que fomentan la interacción y los vínculos sociales (software social). Este apartado tiene una construcción tipo Likert con cinco opciones de respuesta:

- N (ninguna): no tengo ni idea acerca de ello.

- $\mathbf{P}$ (principiante): tengo algún conocimiento al respecto.

- I (intermedio): puedo buscar etiquetas y comentarios.

- A (avanzado): tengo una cuenta propia y contribuyo con publicaciones, archivos y recursos.

- E (experto): tengo un gran dominio de esta herramienta.

\section{(...) los alumnos conocen las \\ funcionalidades básicas de los \\ blogs, pero no hacen uso de esta \\ herramienta como autores propios \\ de un blog}

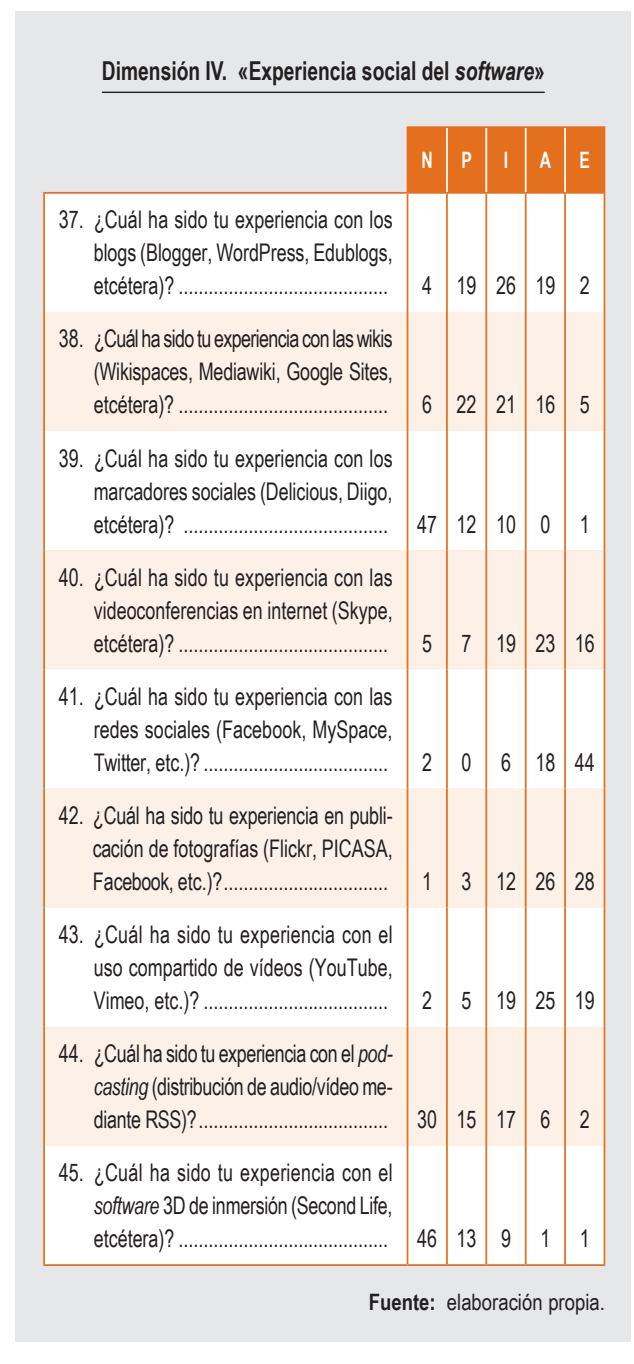

En primer lugar, la pregunta 37 está vinculada a la experiencia de los estudiantes con los blogs. Tal y como indican algunos autores, un blog es un sitio web donde una persona 0 un grupo de personas escriben artículos, comparten vídeos, presentaciones, enlaces, fotos, noticias y reciben comentarios y opiniones de los lectores. Algunos de los servicios gratuitos que nos permiten gestionar un blog son Blogger, WordPress y Edublogs.

En concreto, la opción con un mayor número de respuestas por parte de los encuestados es la de nivel «intermedio» (37\%). Este hecho muestra que los 
alumnos conocen las funcionalidades básicas de los blogs, pero no hacen uso de esta herramienta como autores propios de un blog. Sin embargo, sí que acceden a ellos para obtener información, la cual pueden conseguir a través del sistema de etiquetado de las entradas publicadas (tags).

Por otra parte, la pregunta 38 indaga sobre la experiencia de los participantes con las wikis. Cabe destacar que, según algunos autores, una wiki es un sitio web cuyas páginas pueden ser editadas por múltiples voluntarios a través del navegador web. Los usuarios pueden crear, modificar o borrar un mismo texto que comparten. Los textos 0 «páginas wiki» tienen títulos únicos. Estos autores también indican que «la aplicación de mayor peso y a la que le debe su mayor fama hasta el momento ha sido la creación de enciclopedias colectivas, género al que pertenece la Wikipedia». No obstante, existen una gran variedad de servicios de este tipo, tales como Wikispaces, Mediawiki y Google Sites.

En este caso, la cantidad de respuestas en las opciones «principiante» e «intermedio» es casi la misma, llegando al $31 \%$ y $30 \%$, respectivamente, del total de los encuestados. En consecuencia, los resultados señalan que los alumnos conocen las características básicas de las wikis y acceden a estas herramientas para buscar algún tipo de información. Sin embargo, no disponen de una cuenta en estos servicios y no contribuyen con la creación de sus propias páginas web.

En cuanto la pregunta 39 , esta trata de conocer la experiencia de los estudiantes en relación a los marcadores sociales. Tal y como especifican algunos autores, los marcadores sociales son un tipo de medio social que permite almacenar, clasificar y compartir enlaces en internet o en una intranet. Así pues, en un sistema de marcadores sociales los usuarios guardan una lista de recursos de internet que consideran útiles en un servidor compartido. Otras personas con intereses similares pueden ver los enlaces por categorías, etiquetas 0 al azar. Algunos servicios de marcadores sociales son Delicious y Diigo. Concretamente, las respuestas a esta pregunta son realmente significativas, puesto que el $67 \%$ de los encuestados señalan la opción de «ninguna» experiencia. Por tanto, los alumnos ni conocen las características de estos servicios, ni hacen uso de ellos.
En otro orden de cosas, el ítem 40 está destinado a conocer la experiencia de los encuestados en los servicios de videoconferencias. En este sentido, algunos autores definen la videoconferencia o videollamada como la comunicación simultánea bidireccional de audio y vídeo que permite mantener reuniones con grupos de personas situadas en lugares alejados entre sí. Además, algunas de las facilidades de estos servicios son el intercambio de gráficos, de imágenes fijas, la transmisión de ficheros desde el ordenador, etc. Entre los servicios de este tipo, los que sobresalen son Skype y Hangout.

En este caso, es importante mencionar que la mayoría de los alumnos disponen de las habilidades necesarias para usar los servicios de videoconferencias, ya que las opciones con un mayor número de respuestas son los niveles «intermedio», «avanzado» y «experto» $(27 \%, 33 \%$ y $23 \%$, respectivamente). Así pues, no solo los alumnos conocen las características básicas de estos servicios, sino que también poseen una cuenta en ellos y hacen uso de sus funcionalidades más avanzadas, como, por ejemplo, enviar mensajes instantáneos, adjuntar archivos, compartir la pantalla con otros usuarios, etc.

Otro de los servicios que se valora en este cuestionario son las redes sociales (item 41). Cabe resaltar que, según algunos autores, los servicios de red social están ocupando un lugar destacado en las relaciones humanas medidas tecnológicamente, ya que permiten crear un perfil público, crear una lista de personas con las que se mantiene algún tipo de conexión y gestionar una lista de contactos virtual. Las redes sociales con mayor popularidad son Facebook, Twitter, Google+ y Linkedln (orientada al networking).

(...) existen servicios cuyo objetivo es editar, almacenar y compartir vídeos. Algunos de los servicios con un mayor número de usuarios son YouTube y Vimeo. (...) los alumnos afirman tener un dominio tecnológico de estos servicios 
En concreto, esta pregunta es la única en la que las opciones "experto» y "avanzado» llegan al $89 \%$ de los encuestados (63\% y $26 \%$, respectivamente). En consecuencia, la gran mayoría de los alumnos poseen una cuenta en alguno de los servicios de redes sociales. Asimismo, los discentes están activos en dichos servicios, ya que redactan publicaciones, comparten fotografías, crean eventos, forman parte de grupos de usuarios, etc.

En relación con las redes sociales, hay que hacer referencia a los servicios de publicación de fotografías (pregunta 42 del cuestionario). En líneas generales, estos servicios permiten almacenar y compartir fotografías en línea. Por tanto, algunas redes sociales pueden considerarse, al mismo tiempo, servicios de publicación de fotografías. En este sentido, los servicios de este tipo más destacados son Flickr, PICASA, Instagram y Facebook.

En este caso, los resultados son similares a los de la pregunta anterior, ya que las opciones "experto»y «avanzado» son seleccionadas por el $77 \%$ de los participantes ( $40 \%$ y $37 \%$, respectivamente). Así pues, los alumnos tienen grandes habilidades para publicar fotografías a través de la red, así como para retocarlas/editarlas y compartirlas con otros usuarios.

La pregunta 43 pretende conocer la experiencia de los estudiantes respecto a la compartición de vídeos. En este sentido, existen servicios cuyo objetivo es editar, almacenar y compartir vídeos. Algunos de los servicios con un mayor número de usuarios son YouTube y Vimeo. Cabe precisar que los alumnos afirman tener un dominio tecnológico de estos servicios, ya que las respuestas más frecuentes son «intermedio», "avanzado» y «experto» (los porcentajes son $27 \%, 36 \%$ y $27 \%$, respectivamente). Por tanto, estos resultados quieren decir que los discentes están registrados en estos servicios y que, además, hacen uso de ellos a través de la edición y compartición de vídeos de manera privada o pública.

A continuación, el propósito del ítem 44 es saber la experiencia de los encuestados en torno a los servicios de podcasting. De manera general, Sellas (2011) resume el concepto de podcast como «un archivo sonoro que podemos descargar de internet de forma automática mediante un sistema de suscripción, y sincronizarlo con un reproductor digital portátil». De una forma más detallada, Laaser, Liliana y Rodríguez (2010) explican el funcionamiento del podcasting:

«El podcaster graba y edita el podcast, con el uso de un micrófono y un editor de sonido, [y] lo guarda como archivo MP3. Luego sube este archivo a un sitio web reservado para los podcasts junto con un archivo de XML que describe la dirección donde se ubica el podcast, quién es el autor y cómo se llama el archivo. Cuando hay una secuencia se habla de episodios. Para escuchar el podcast el usuario necesita un feedreader o podcatcher que está activado a través de una subscripción del sitio web».

Algunos de estos servicios son Podcast y Spreaker. En concreto, resulta significativo que el $43 \%$ de los estudiantes señale la opción «ninguna» en este tipo de servicios, por lo que no conocen qué es el podcasting, $\mathrm{n}$ tampoco las funcionalidades básicas de estos servicios.

Finalmente, se analiza la experiencia de los participantes en relación al software 3D de inmersión (pregunta 45). Este tipo de espacios son definidos por Sicilia y García (2012) como «simulaciones sobre el ordenador que tienen una interfaz en tercera dimensión para dar a sus usuarios la sensación de inmersión total en todas sus interacciones con el ambiente». Existen muchos mundos virtuales en internet (World of Warcraft, There, Entropía Universe), pero el más grande y representativo es Second Life. Según Sicilia y García (2012), Second Life «es un mundo virtual on-line creado por Linden Labs, conectado con la economía real, que cuenta con más de 13 millones de usuarios registrados que tienen allí sus propiedades». Así pues, estos autores prosiguen que «los residentes de Second Life diseñan, conducen negocios, construyen relaciones, se entretienen, aprenden y viven sus vidas virtuales». Sin embargo, los resultados de la encuesta revelan que los alumnos no conocen las funcionalidades de este tipo de servicios, puesto que la opción que sobresale respecto a las demás es «ninguna» experiencia $(66 \%)$.

Una vez descritos los resultados obtenidos en la dimensión IV, hay que mencionar que guardan relación con los datos de la investigación de Cabero y Marín (2014), realizada en diversas ciudades españolas. Según estos autores: 
«En lo que se refiere a la experiencia que los alumnos tienen respecto al software social, encontramos tres niveles de formación. Por una parte, estarían las tecnologías en la que nos señalan tener un "avanzado" nivel de formación: las redes sociales (49,3\%), la publicación de fotografías $(45,8 \%)$ y compartir vídeos $(31,4 \%)$; $y$, por otro, las tecnologías donde su nivel de formación mayoritario es "ninguno" o "principiante": wikis (34,1\%, "principiante"), marcadores sociales $(71,8 \%$, "ninguna"), podcasting (69,8\%, "ninguna") y experiencias en software en $3 \mathrm{D}$ de inmersión (84,1\%, "ninguna"). Finalmente, encontramos algunos casos donde las opciones "principiante" y "avanzada" ocupan porcentajes muy similares: blog $(34,1 \%$ y $28,8 \%$ y videoconferencia a través de la web $(34,7 \%$ y $20,7 \%)$. Hay que destacar que el caso de las redes sociales es el único de esta parte del cuestionario donde la suma de las opciones "avanzado" y "experto" llegan al 50\%».

Por último, las respuestas de este cuestionario también concuerdan con los resultados alcanzados por Cabero y Marín (2013) en la investigación que realizaron de Sudamérica y España. En este sentido, los autores apuntan:

«Los datos encontrados permiten constituir tres grupos según el nivel de formación que afirman tener los alumnos. Por una parte, están las tecnologías que señalan que tienen un nivel de formación "avanzado": redes sociales $(46,93 \%)$ y la publicación de fotografías (42,97\%); por otra, las tecnologías en las que su nivel era "ninguno": wikis $(32,84 \%)$, marcadores sociales $(71,74 \%)$, podcasting $(67,29 \%)$ y experiencias en software 3D de inmersión (77,40\%). Finalmente, hay algunos casos en los que

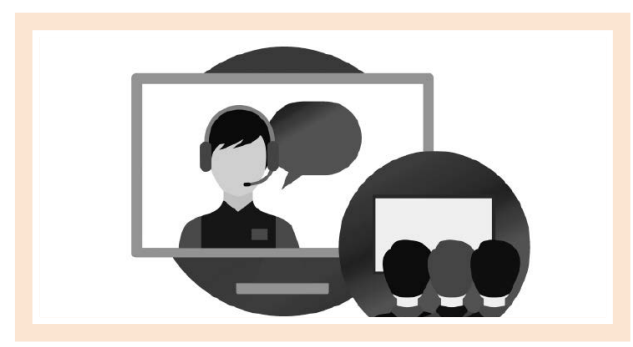

\section{(...) el $43 \%$ de los estudiantes (...) no conocen qué es el podcasting, ni tampoco las funcionalidades básicas de estos servicios}

las opciones "principiante" e "intermedio" ocupan porcentajes similares: blog $(32,21 \%)$ y videoconferencia a través de la web $(30,79 \%)$.

Esos datos no nos permiten señalar la fuerte capacitación de los alumnos en determinadas tecnologías, aunque sí destacan en lo que se refiere a la publicación de fotografías y a compartir vídeos, en los que consideraban que tenían una formación "intermedia" y "avanzada". Destaca el nivel "avanzado" y "experto" de su experiencia con las redes sociales $(77,15 \%)$ ».

\section{Dimensión V. «Software social para el aprendizaje»}

Está configurada para saber el grado de interés de los estudiantes en relación al manejo y al uso del software social para el aprendizaje. Este apartado tiene una construcción tipo Likert con cinco opciones de respuesta:

- NN (nada de nada interesado).

- NI (no muy interesado).

- N (no lo sé).

- I (interesado).

- MI (muy interesado).

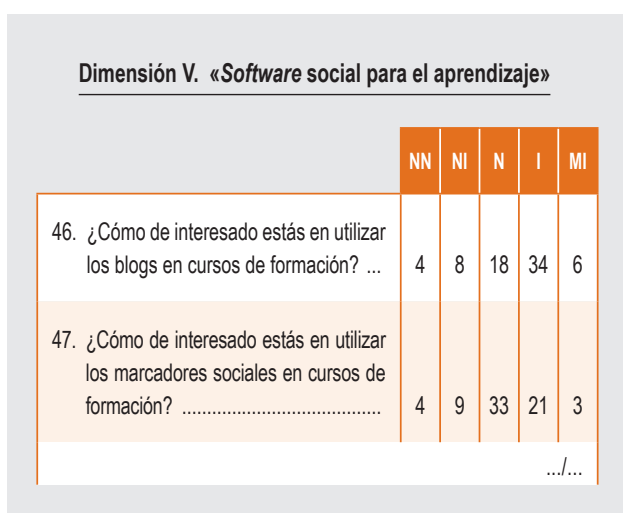




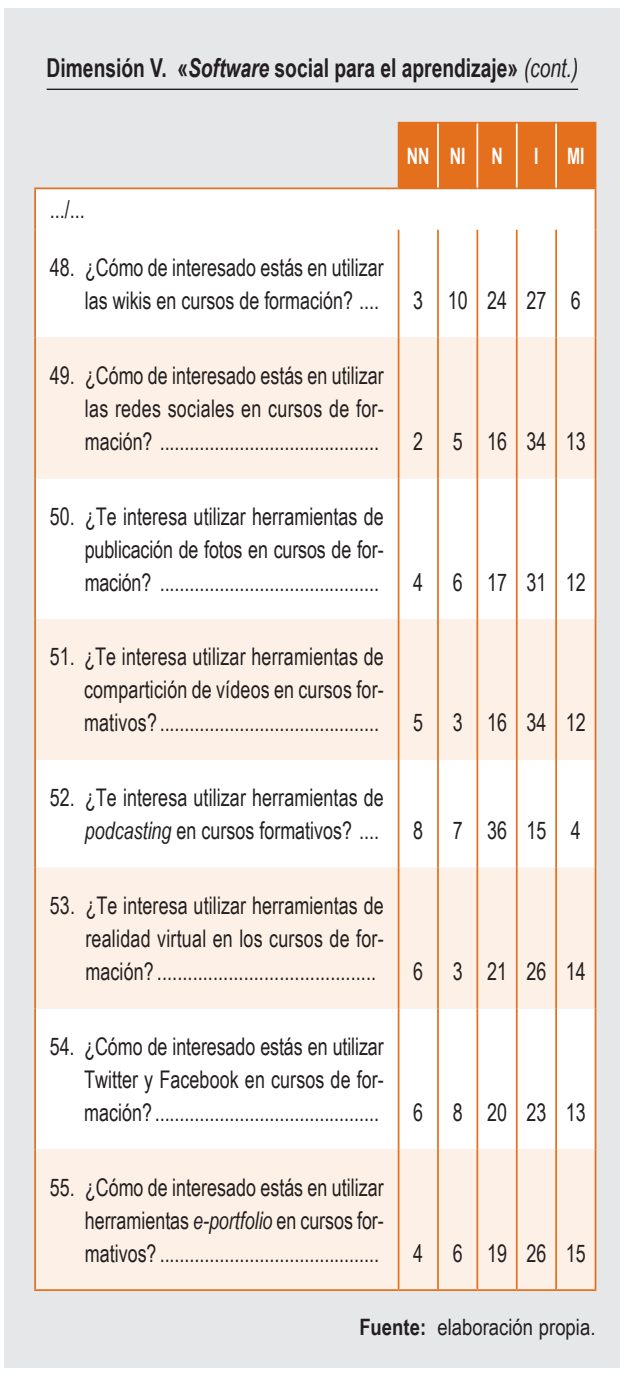

En primer lugar, la pregunta 46 está enfocada para conocer el grado de interés de los participantes por utilizar los blogs en cursos de formación. Cabe destacar que la creación de un blog constituye una experiencia educativa en la que sus usuarios realizan constantemente actividades de aprendizaje. Algunas de estas actividades han sido recogidas por Contreras (2004):

«(...) a) producen textos; b) leen y analizan textos; c) buscan, seleccionan e intercambian información; d) opinan o guardan silencio; e) reflexionan y evalúan los acontecimientos de su vida, de su grupo, de su nación o del mundo; f) toman decisiones y posturas; g) defienden ideologías; h) comparten sentimientos, intereses, ideas, etc.; i) forman redes».

En concreto, la opción con una mayor cantidad de respuestas es «interesado», la cual ha sido seleccionada por el $48 \%$ de los encuestados. Además, si se juntan las respuestas de las opciones «interesado» $(48 \%)$ y «muy interesado» (9\%), resulta significativo que más de la mitad de los estudiantes quieren introducir estos servicios en sus cursos académicos (en total el $57 \%$ ).

A continuación, el ítem 47 pretende conocer el grado de interés de los encuestados sobre el uso de marcadores sociales en cursos de formación. Así pues, el principal uso didáctico que se le puede dar a este tipo de servicios es organizar recursos web referentes a una asignatura o trabajo de investigación. La opción con un mayor número de respuestas es «no lo sé» $(47 \%)$. Entre las opciones «nada de nada interesado», «no muy interesado» y «no lo sé» se encuentran más de la mitad de los participantes $(6 \%, 13 \%$ y $47 \%$, respectivamente). Por tanto, los resultados no muestran un claro interés por utilizar marcadores sociales en cursos académicos.

Por otra parte, la pregunta 48 está relacionada con el grado de interés de los participantes para utilizar las wikis en cursos de formación. Hay que hacer referencia a Lott (2005), citado por Adell (2007), quien recoge algunos de los usos de las wikis en educación:
«(...) a) espacio de comunicación de la clase; b) espacio de colaboración de la clase/base de conocimientos; c) espacio para realizar y presentar tareas/portafolios electrónicos; d) archivo de textos en proceso de elaboración; e) manual de la clase/autoría colaborativa; f) espacios para los proyectos en grupo».

A pesar de la gran cantidad de usos didácticos que poseen las wikis, los resultados muestran que la mayoría de los estudiantes no están interesados en utilizar este tipo de servicios en cursos académicos. En este sentido, la opción con más cantidad de respuestas es «interesados» (39\%). Entre las opciones «nada de nada interesado», «no muy interesado» y «no lo 
sé» se encuentran más de la mitad de los participantes (4\%, $14 \%$ y $34 \%$, respectivamente).

La siguiente pregunta es la número 49 , la cual está referida al interés de los encuestados por el uso de las redes sociales en cursos de formación. Tal y como se ha señalado antes, algunas de las ventajas de las redes sociales en el contexto académico son (Gómez, Roses y Farias, 2012):

«Las redes permiten y favorecen publicar y compartir información, el autoaprendizaje; el trabajo en equipo; la comunicación, tanto entre alumnos como entre alumno-profesor; la retroalimentación; el acceso a otras fuentes de información que apoyan e incluso facilitan el aprendizaje constructivista y el aprendizaje colaborativo; y el contacto con expertos».

En este caso, cabe resaltar la actitud positiva de los alumnos respecto a las redes sociales, ya que más de la mitad de ellos están «interesados» 0 «muy interesados» (54\% y $10 \%$, respectivamente) en introducir este tipo de servicios en el ámbito académico.

Seguidamente, el ítem 50 trata de conocer el interés de los encuestados por introducir la publicación de fotografías en los cursos de formación que realizan. Algunos de los usos didácticos que pueden tener estos servicios son los siguientes: compartir fotografías del trabajo diario, seleccionar fotografías con distintos tipos de licencia para incluirlas en trabajos de investigación, editar fotografias y posteriormente publicarlas, etc. En este sentido, las respuestas de los participantes indican que están interesados en usar este tipo de servicios, ya que más de la mitad de los estudiantes han seleccionado las opciones «interesado» y «muy interesado» (44\% y $17 \%$, respectivamente).

Por otra parte, la pregunta 51 tiene como objetivo conocer el interés de los alumnos por utilizar servicios de compartición de vídeos en cursos de formación. Cabe indicar que estos servicios pueden ser útiles en educación para buscar y compartir vídeos que traten de alguna información concreta, seguir canales de vídeos de interés, editar vídeos como parte de una actividad de aprendizaje, etc. En concreto, la opción con mayor cantidad de respuestas es «interesado» (49\%), seguida de «no lo sé» (23\%) y «muy interesado»
(17\%). Por tanto, los estudiantes muestran una actitud receptiva para emplear dichos servicios en cursos académicos.

En otro orden de cosas, el ítem 52 sirve para saber el interés de los encuestados por usar herramientas de podcasting en cursos de formación. Algunos usos didácticos de este tipo de servicios son recogidos por Laaser, Liliana y Rodríguez (2010):

«Los podcasts pueden emplearse, por ejemplo, como libro auditivo. Este formato transforma la experiencia de lectura en una escucha de la misma. Se puede grabar la lectura de un documento para usarlo auditivamente (empleo altamente recomendable para personas con discapacidades visuales). También pueden utilizarse en programas de entrevistas, ya que son una excelente herramienta para la transmisión de las mismas. Son útiles, además, para la grabación de ponencias, ya que se pueden archivar y emplear como material de ayuda memoria. Su uso en la educación a distancia se evidencia en el apoyo al aprendizaje autónomo, en los comentarios para las tareas a enviar, en la preparación para exámenes, etc.».

A pesar de los beneficios pedagógicos mencionados, la opción más escogida en el cuestionario administrado es «no lo sé» (52\%). Por tanto, se presupone que el alumnado no sabe si está interesado en utilizar podcasting en el contexto educativo, hecho que puede ser debido a que los estudiantes no son conscientes de sus ventajas.

En cuanto a las herramientas de realidad virtual, la pregunta 53 trata de conocer el interés de los participantes por utilizar dichos servicios en los cursos de formación. Entre las aplicaciones educativas de los mundos virtuales se pueden detallar las siguientes ( $\mathrm{Si}$ cilia y García, 2012):

«(...) a) simulaciones; b) colaboración; c) proyectos de negocio con clientes reales; d) juego de roles; e) trabajar en clases virtuales; f) construir ambientes colaborativamente; g) interpretar, analizar, descubrir, evaluar y resolver problemas». 
En concreto, los estudiantes muestran una actitud positiva hacia el uso de los softwares 3D de inmersión, ya que más de la mitad de los encuestados han seleccionado las opciones «muy interesado»e «interesado» (20\% y $37 \%$, respectivamente). En consecuencia, los alumnos quieren incluir estas herramientas en su contexto académico.

En lo que respecta al ítem 54, hay que resaltar que retoma el tema de las redes sociales (abordado en el ítem 49) para profundizar en el interés por utilizar en el ámbito educativo los dos servicios de redes sociales más representativos: Facebook y Twitter. A pesar de que estas herramientas no han sido creadas con fines educativos, sus usos en este contexto son múltiples: compartir enlaces con un grupo, interactuar con miembros de un grupo, consultar opiniones del grupo, coordinar el estudio grupal o dar información de la clase (Gómez y López, 2010).

En este sentido, la opción con un mayor número de respuestas es «interesado» (33\%), seguida de «no lo sé» $(29 \%)$ y «muy interesado» (19\%). Por tanto, se puede percibir una tendencia positiva por utilizar Facebook y Twitter en educación y, así, vincular la vida personal/privada con la profesional.

En último lugar, la pregunta 55 está relacionada con el interés de los participantes por usar herramientas e-portfolio en cursos formativos. Algunas de las ventajas de utilizar e-portfolios en educación son expuestas por Zubizarreta (2009), citado por Cabero y Marín (2015):

\begin{abstract}
«(...) a) desarrollo del aprendizaje activo; b) motivan a los estudiantes; $c$ ) son instrumentos que favorecen el feedback; d) son instrumentos de debate del desempeño de los estudiantes; e) son una exposición "referencia" de rendimiento; f) son accesibles; g) pueden almacenar múltiples medios de comunicación; h) son fáciles de actualizar; i) permiten una remisión de trabajos de alumnos».
\end{abstract}

Así pues, los alumnos están interesados en utilizar herramientas e-portfolio en los cursos académicos que llevan a cabo, ya que la opción con un mayor número de respuestas es «interesado» (37\%). Asimismo, la opción «muy interesado» también ha sido escogida por un gran número de encuestados $(21 \%)$.
Tras analizar la dimensión $\mathrm{V}$, hay que tener en cuenta que los resultados expuestos están en consonancia con los recogidos por Cabero y Marín (2014) en su investigación realizada en diversas ciudades españolas. En este sentido, según exponen los autores:

«Los resultados encontrados son un poco contradictorios, ya que, por una parte, indican que la opción más seleccionada en todos los casos ha sido la de "interesado", pero tenemos que señalar que en dos de ellas, "¿Cómo de interesado estás en utilizar los marcadores sociales que se emplean en los cursos de formación que realizas? (por ejemplo, Delicious, Diigo)" y "¿Cómo de interesado estás en contar con herramientas de podcasting utilizados en los cursos de formación que realizas?", los porcentajes de respuesta en la opción "no muy interesado" eran en cierta medida elevados: $32,4 \%$ y $28,1 \%$, respectivamente, aunque el porcentaje alcanzado en la opción "no lo sé" era, en el primero de los casos, del $17,0 \%$ y $19,7 \%$ respectivamente, lo que sugiere un cierto desconocimiento de los alumnos de estas tecnologías».

Finalmente, cabe mencionar que la similitud de las respuestas también es notable en la investigación anterior de Cabero y Marín (2013). Así pues, las percepciones de los autores son las siguientes:

«La opción más seleccionada ha sido "interesado". Sin embargo, en tres ítems - “En qué medida te interesa utilizar los marcadores sociales que se emplean en los cursos de formación que realizas?" (19,52\%), "¿En qué medida te interesa disponer de las herramientas de podcasting que se utilizan en los cursos de formación que realizas?" $(22,34 \%)$ y "¿En qué medida te interesa tener las herramientas tipo e-portfolio que se utilizan en los cursos de formación que realizas?" (18,67\%)-, los porcentajes de respuesta en la opción "no muy interesado" fueron elevados». 


\section{CONCLUSIONES Y TRABAJO FUTURO}

\subsection{Conclusiones}

Las conclusiones de dicha investigación se estructuran en torno a las cinco dimensiones analizadas. En este sentido, en la dimensión I («Datos descriptivos») se concluye que la mayoría de los estudiantes del grado en Educación Infantil en la Universidad de Alicante son mujeres. Además, los alumnos universitarios actualmente cuentan con un ordenador personal, así como con acceso a internet. También, cabe destacar que los discentes acceden de forma regular a internet con fines educativos.

A continuación, en la dimensión II («Preferencias de aprendizaje»), se puede concluir que los alumnos muestran una actitud receptiva al desarrollo del trabajo colaborativo, ya que tienen en cuenta los beneficios de este tipo de aprendizaje: rápida creación de ideas, ayuda a los miembros del equipo, desarrollo del compañerismo y adquisición de habilidades sociales.

En cuanto a la dimensión III («Habilidades tecnológicas»), hay que concluir que los alumnos disponen de una buena actitud para el uso de ordenadores, así como de habilidades técnicas básicas para utilizarlos. Asimismo, se concluye que los estudiantes presentan un mayor dominio de herramientas y programas como procesadores de texto, presentaciones de diapositivas, antivirus, motores de búsqueda y herramientas de comunicación. Sin embargo, los discentes no se muestran tan capacitados para realizar hojas de cálculo y administrar directorios de archivos.

En relación a la dimensión IV («Experiencia social del software»), se concluye que los estudiantes tienen grandes habilidades para utilizar algunos servicios, pero presentan carencias en el manejo de otras herramientas. En este sentido, los alumnos tienen un nivel avanzado o experto en los siguientes servicios: videoconferencias, redes sociales, publicación de fotos y compartición de vídeos. Por otra parte, los discentes tienen un nivel de experiencia intermedio en las siguientes herramientas: blogs y wikis. Finalmente, los estudiantes no tienen ninguna experiencia y, por tanto, no disponen de habilidades para usar los siguientes servicios: marcadores sociales, podcasting y software 3D de inmersión.

Por último, en la dimensión V («Software social para el aprendizaje») se concluye que los alumnos no se muestran interesados en utilizar todo tipo de herramientas en el contexto educativo. Así pues, las herramientas en las que tienen un interés significativo son las siguientes: blogs, redes sociales, herramientas de publicación de fotos, herramientas de compartición de vídeos, software 3D de inmersión y servicios e-portfolios. Sin embargo, las herramientas que los estudiantes no quieren o no saben si quieren introducir en el ámbito académico se especifican a continuación: marcadores sociales, wikis y podcasting.

Teniendo en cuenta las conclusiones alcanzadas en cada una de las dimensiones expuestas, es importante mencionar las conclusiones generales a las que se han llegado a través de este trabajo de investigación:

- Los alumnos muestran altas percepciones y una actitud positivista para trabajar en grupo y de manera colaborativa.

- Los estudiantes no son tan competentes en el manejo de las nuevas tecnologías como el profesorado puede pensar, ya que su nivel de experiencia varía de unas herramientas a otras.

- Los discentes están interesados en aprender a manejar ciertas herramientas tecnológicas pertenecientes a los social media y a introducirlas en el ámbito educativo.

En consecuencia, la hipótesis de la cual se partía al comienzo de este trabajo ha sido verificada, ya que «los alumnos del grado de Educación Infantil de la Universidad de Alicante tienen actitudes positivas respecto al trabajo colaborativo en las redes sociales».

\subsection{Trabajo futuro}

Finalmente, cabe destacar cómo se podría continuar el presente trabajo de investigación. En este sentido, dado que dicha investigación se centra en analizar las apreciaciones respecto al trabajo colaborativo y las redes sociales del alumnado del grado en Educación Infantil de la Universidad de Alicante, una propuesta adecuada de trabajo futuro sería llevar a cabo el mismo estudio en las universidades de Valencia y Castellón. De esta manera, analizando los resultados de los tres trabajos se podrían extraer unas conclusiones generales respecto al trabajo colaborativo y las redes sociales a nivel autonómico, en este caso, la Comunidad Valenciana.

Asimismo, teniendo en cuenta el presente estudio realizado con los alumnos de la Universidad de Alicante y la 
investigación de Cabero y Marín (2014) llevada a cabo en universidades de otras ciudades españolas (Sevilla, Huelva, Córdoba y País Vasco), otra propuesta de trabajo futuro sería continuar diagnosticando las percepciones de los alumnos del resto de universidades de España. En consecuencia, los resultados que se alcancen permitirían conocer las actitudes del estudiantado respecto a esta temática a nivel estatal. En este sentido, dichas conclusiones brindarían la oportunidad

\section{BIBLIOGRAFÍA}

Adell, J. [2007]: «Wikis en educación», en J. Cabero (ed.), Nuevas tecnologías aplicadas a la educación, Madrid: McGraw-Hill, págs. 323-333.

Bicen, H. y Cavus, N. [2011]: «Social network sites usage habits of undergraduate students: case study of Facebook», Procedia-Social and Behavioral Sciences, 28, págs. 943-947.

Cabero, J. [2009]: «Educación 2.0. ¿Marca, moda o nueva visión de la educación?», en C. Castaño (ed.), Web 2.0. El uso de la web en la sociedad del conocimiento, investigación e implicaciones educativas, Caracas: Universidad Metropolitana, págs. 13-34.

[2011]: Tendencias educativas para el siglo XXI, Madrid: Centro de Estudios Financieros.

Cabero, J. y Marín, V. [2013]: «Percepciones de los estudiantes universitarios latinoamericanos sobre las redes sociales y el trabajo en grupo», Revista de Universidad y Sociedad del Conocimiento (RUSC), 10 (2), págs. 219-235.

[2014]: «Posibilidades educativas de las redes sociales y el trabajo en grupo. Percepciones de los alumnos universitarios», Comunicar: Revista Científica de Comunicación y Educación, 21 (42), págs. 165-172.

Contreras, F. [2004]: «Weblogs en educación», Revista Digital Universitaria, 5 (10), págs. 1-12.

Espuny, C.; González, J.; Lleixà, M. y Gisbert, M. [2011]: "Actitudes y expectativas del uso educativo de las redes sociales en los alumnos universitarios», Revista de Universidad y Sociedad del Conocimiento (RUSC), 8 (1), págs. 171-185.

Fernández, T. [2013]: «Aprendizaje colaborativo y uso de las redes sociales en educación primaria», Didáctica: Lengua y Literatura, 25, págs. 157-187. de realizar una comparativa entre los alumnos de diferentes universidades españolas, así como de comparar las percepciones de alumnos españoles y extranjeros.

En última instancia, es importante mencionar que este trabajo, basado en los estudios de Cabero y Marín (2013 y 2014), aporta un instrumento útil -la encuestapara diagnosticar las percepciones del alumnado en relación al trabajo en grupo y las redes sociales.

García, A. [2009]: «Las redes sociales como herramientas para el aprendizaje colaborativo: una experiencia con Facebook», Re-Presentaciones: Periodismo, Comunicación y Sociedad, 5, págs. 48-63.

García, S. A. y Alonso, M. ${ }^{a}$ M. [2014]: «Las redes sociales en las universidades españolas», Vivat Academia, 17 (126), págs. 54.

Garrigós, I.; Mazón, J. N.; Saquete, E.; Puchol, M. y Moreda, P. [2010]: «La influencia de las redes sociales en el aprendizaje colaborativo», XVI Jornadas de Enseñanza Universitaria de la Informática, págs. 531-534.

Gómez, M. ${ }^{a}$ T. y López, N. [2010]: «Uso de Facebook para actividades académicas colaborativas en educación media y universitaria», Signos Universitarios Virtual, 8 (9).

Gómez, M.; Roses, S. y Farias, P. [2012]: «El uso académico de las redes sociales en universitarios», Comunicar, 19 (38), págs. 131-138.

Haro, J. J. de [2010]: Redes sociales para educación, Madrid: Anaya.

Laaser, W.; Liliana, S. y Rodríguez, L. C. [2010]: «Podcasting: ¿un nuevo medio para la educación a distancia?», Revista de Educación a Distancia (RED), 23.

Martínez, M. ${ }^{a}$ Y. [2014]: «Redes sociales y TIC, su papel en la educación superior del siglo XXI», Historia y Comunicación Social, 19, págs. 63-71.

Poellhuber, B.; Anderson, T. y Roy, N. [2011]: «Distance students' readiness for social media and collaboration», The International Review of Research in Open and Distributed Learning, 12 (6), págs. 102-125. 


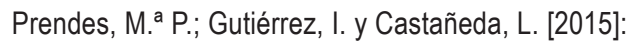
«Perfiles de uso de redes sociales: estudio descriptivo con alumnado de la Universidad de Murcia», Revista Complutense de Educación, 26, págs. 175-195.

Rendón, L. M. ${ }^{\text {a } ~}$ Ortega, J. A. [2015]: «Aprendizaje colaborativo mediante redes sociales y radio comunitaria Web 2.0», Revista Lasallista de Investigación, 12 (2), págs. 54-65.

Sánchez, J.; Ruiz, J. y Sánchez, E. [2015]: «Uso problemático de las redes sociales en estudiantes universitarios», Revista Complutense de Educación, 26, págs. 159-174.
Sellas, A. [2011]: El podcasting: la (R) evolución sonora, Barcelona: Editorial Universitat Oberta de Catalunya.

Sicilia, M. Á. y García, E. [2012]: Aprendizaje de tecnologías de la información y la comunicación, Madrid: Centro de Estudios Financieros.

Sixto, J. y Túñez, M. [2012]: «Las redes sociales como entorno docente: análisis del uso de Facebook en la docencia universitaria», Pixel-Bit: Revista de Medios y Educación, 41, págs. 77-92.

Towner, T. y Muñoz, C. [2011]: «Facebook and education: a classroom connection», Cutting-Edge Technologies in Higher Education, 1, págs. 33-57.

\section{ANEXO}

\section{Gráficos con los resultados de las preguntas formuladas}

\section{GRÁFICOS CON LOS RESULTADOS DE LAS PREGUNTAS FORMULADAS EN EL CUESTIONARIO}

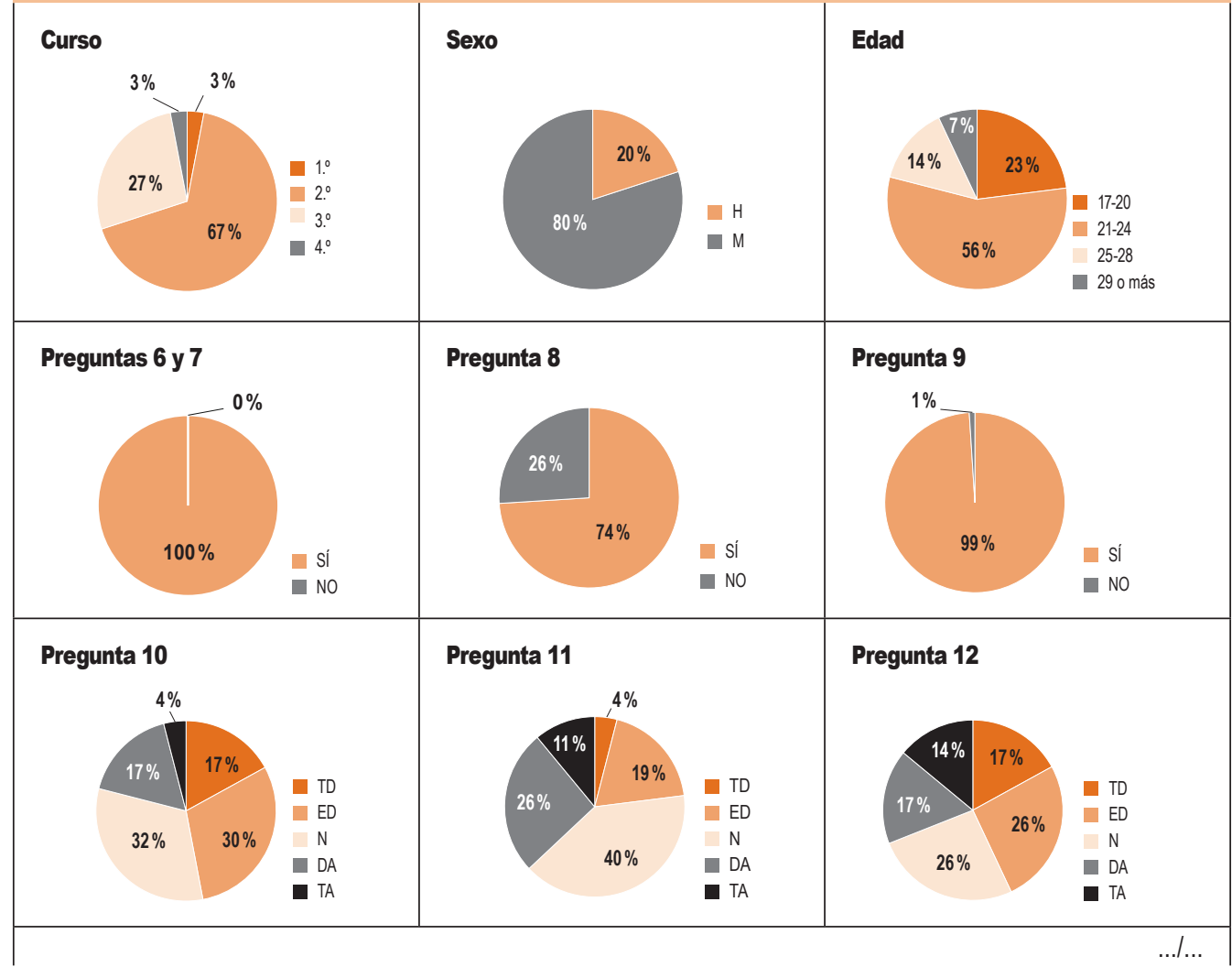




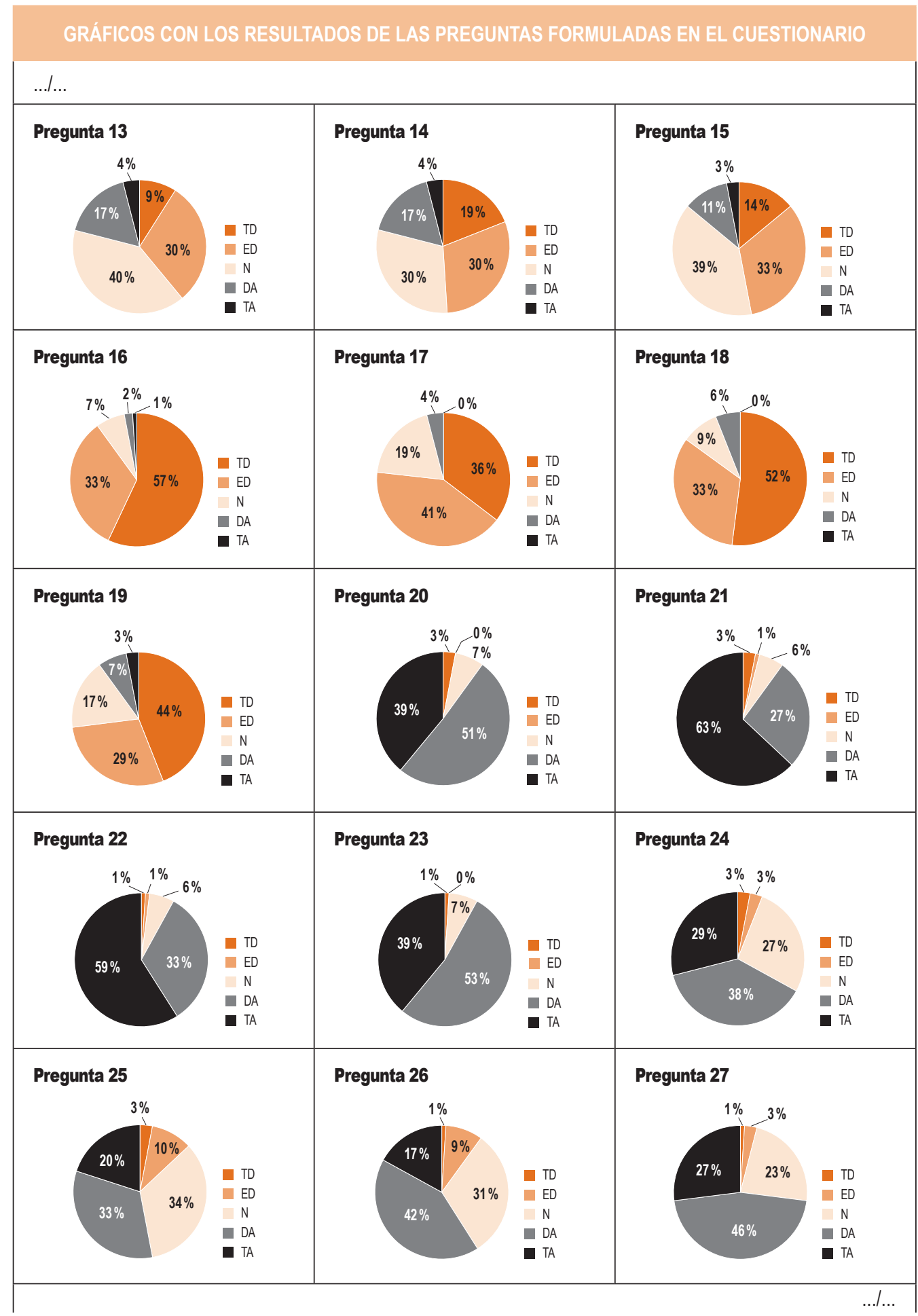




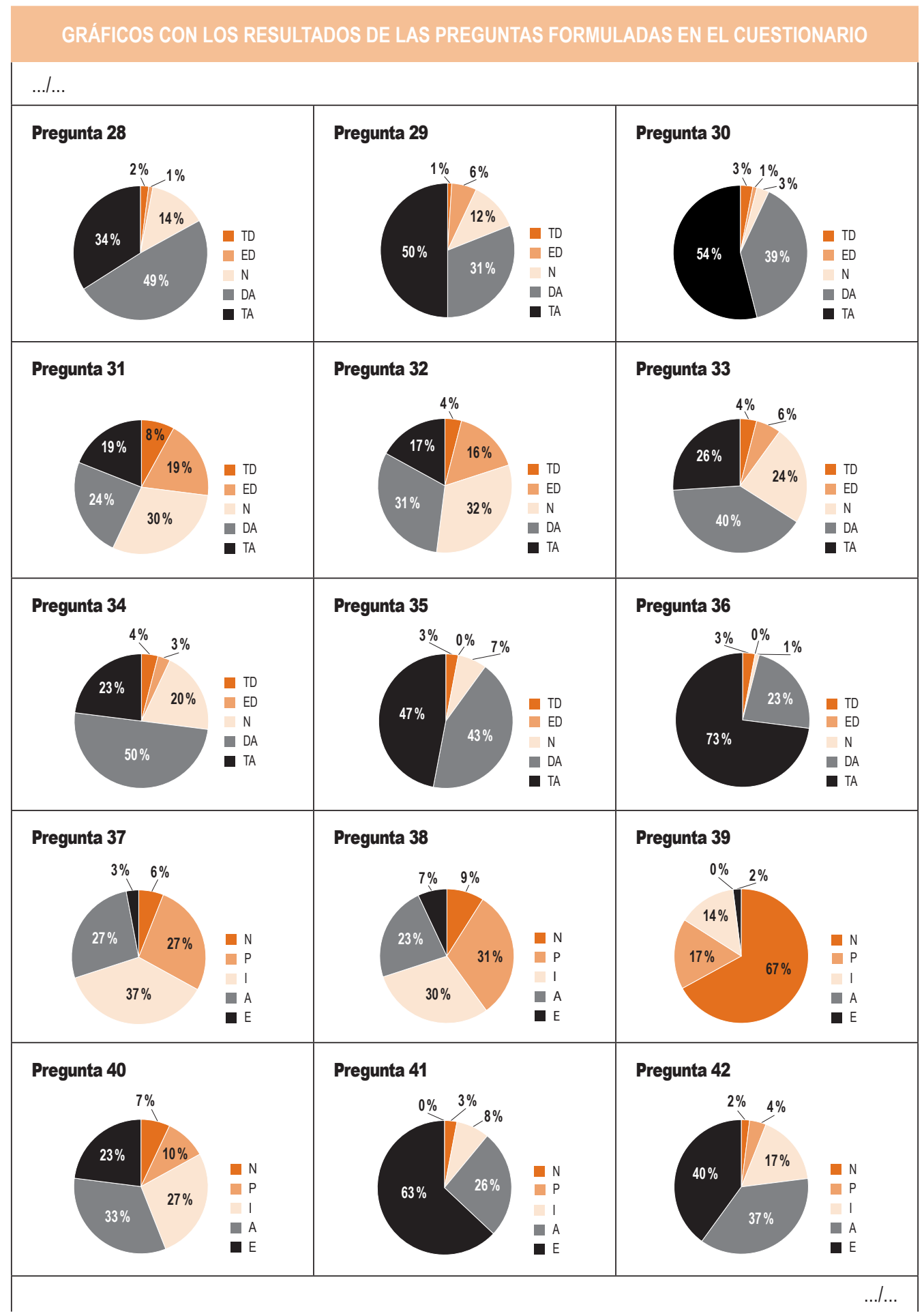




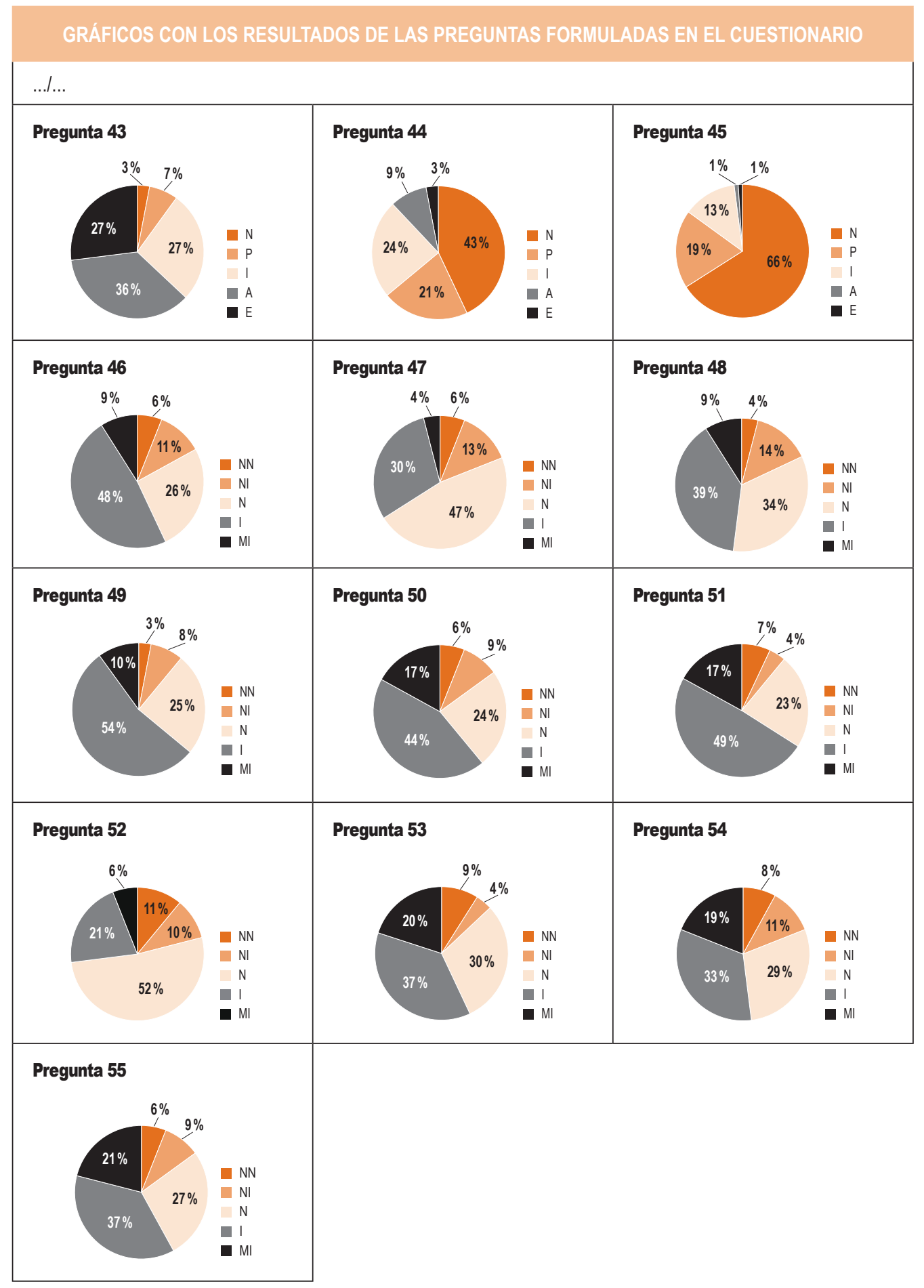

